Uwe Wagschal/Aurel Croissant/Thomas Metz/Christoph Trinn/Nicolas Schwank

\title{
Kulturkonflikte in inner- und zwischenstaatlicher Perspektive
}

Der Beitrag untersucht die Bedeutung kultureller Faktoren (gemessen als sprachliche, religiöse und kulturelle Fraktionalisierung) für das inner- und zwischenstaatliche Konfliktgeschehen weltweit zwischen 1950 und 2005. Ausgehend von einer theoretisch vorgenommenen Trennung zwischen Ursache und Gegenstand von Auseinandersetzungen fragt er erstens, wann Kultur Konflikte auslöst, sowie zweitens, wann Kultur in Form der Identität von Akteuren selbst Thema eines Konflikts ist. Für die erste Frage zeigt sich als nichtlinearer Effekt, dass ein mittlerer Grad sprachlicher Fraktionalisierung das Konfliktrisiko anhebt. Ein ähnliches Muster wird für die religiöse Fraktionalisierung sichtbar, ist aber weniger robust. Zur Beantwortung der zweiten Frage wird die Teilmenge an Konflikten betrachtet, in denen Sprache, Religion und geschichtliche Identitätskonstruktionen eine wesentliche Rolle spielen und das Konfliktgeschehen als Thema kulturell überformen (Kulturkonflikte). Obwohl sich diese kulturell aufgeladenen Konflikte durch ein erhöhtes Gewaltniveau auszeichnen, liefert die statistische Analyse kaum Hinweise auf ein von nicht-kulturellen Konflikten abweichendes Ursachenprofil.

\section{Einleitung}

Der vorliegende Beitrag untersucht die Bedeutung von Kultur für das inner- und zwischenstaatliche Konfliktgeschehen der Jahre 1950 bis 2005. Anders als in bisherigen Ansätzen ${ }^{1}$ wird eine doppelte Perspektive eingenommen: Erstens wird die Bedeutung kultureller Größen als Erklärung für das allgemeine Phänomen Konflikt untersucht. Da Ursache und Gegenstand einer Auseinandersetzung nicht identisch sein müssen, wird zweitens nach den Ursachen von Konflikten gefragt, in denen Kultur ein zentrales Thema der Auseinandersetzung ist. Mit anderen Worten, es wird untersucht, ob und wie gut kulturelle Faktoren das Auftreten politischer Auseinandersetzungen im Allgemeinen und kultureller Konflikte im Spezifischen erklären können. Beide Perspektiven zusammengenommen ergeben eine umfassendere Betrachtung der Beziehung zwischen Kultur und Konflikt als bisher üblich. Die getrennte Betrachtung von inner- und zwischenstaatlichen Konflikten erlaubt zudem eine differenzierte Antwort auf die ursprünglich von Samuel P. Huntington (1993) aufgeworfene und von ihm nur auf zwischenstaatliche Konflikte bezogene These, nach der kulturelle Unterschiede die Triebfeder moderner Kriege seien.

1 Vgl. Huntington (1993; 1997); Russett/Oneal/Cox (2000); Henderson/Trucker (2001); Alesina et al. (2003); Fearon/Laitin (2003); Collier/Hoeffler (2004); Gartzke/Gleditsch (2006). 
Der vorliegende Beitrag gliedert sich wie folgt: Im Anschluss wird ein allgemeiner Begriff für Kulturkonflikte als Untergruppe politischer Auseinandersetzungen entwickelt, in denen Kultur als identitätsstiftende Größe ein bestimmendes Element der Konfrontation darstellt. Danach wird diskutiert, wie kulturelle Konflikte innerhalb des zugrundeliegenden CONIS-Konfliktdatensatzes (CONIS = Conflict Information System) identifiziert werden können und es wird ein deskriptiver Überblick über das weltweite Konfliktgeschehen im Untersuchungszeitraum gegeben. Anschließend wird im analytischen Teil der Arbeit geprüft, (a) wie die kulturelle Fraktionalisierung eines Landes sein Konfliktniveau allgemein beeinflusst und (b) welche Einflussgrößen einen signifikanten Erklärungsbeitrag leisten können, wenn man den Blick auf die Untergruppe der kulturellen Konflikte verengt. Dabei zeigt sich, dass vor allem der Grad an sprachlicher Fraktionalisierung das Konfliktrisiko ansteigen lässt. Für die religiöse Fraktionalisierung ist diese Verbindung weniger stabil. Eine weitere Spezifizierung der Zusammenhänge liefert Hinweise darauf, dass die Beziehung beider Größen zum Konfliktrisiko nichtlinear zu sein scheint. Ein Fazit rundet den Beitrag ab.

\section{Kulturelle Konflikte: Theorie und Empirie}

Im Folgenden werden kulturelle Konflikte als eine von mehreren Unterformen des politischen Konflikts definiert. Politische Konflikte sind Auseinandersetzungen zwischen zwei oder mehr Akteuren über mindestens einen Konfliktgegenstand, die mittels messbarer Maßnahmen ausgetragen werden. ${ }^{2}$ Bei allen Konflikten handelt es sich außerdem um Kommunikationssituationen (vgl. Gurr 1970: 223-224; Messmer 2003), in denen zwischen Kommunikationspartnern (den Konfliktparteien) ein Kommunikationsinhalt (das Thema des Konflikts) über Medien respektive Kommunikationsmittel (die Konfliktmaßnahmen) ausgetauscht wird. Die allgemeine Obergruppe der politischen Konflikte lässt sich in zweifacher Hinsicht unterteilen: Zum einen können Konflikttypen anhand der beteiligten Konfliktparteien in innerstaatliche, zwischenstaatliche und transnationale Konflikte unterschieden werden. Wir gehen davon aus, dass unser Modell der Konfliktkommunikation sowohl für innerstaatliche als auch für zwischenstaatliche Konfliktkonstellationen gilt: Zwar unterscheidet sich die Interaktion zwischen Staaten, zwischen staatlichen und nicht-staatlichen Akteuren sowie zwischen nicht-staatlichen Akteuren untereinander, doch auf dem Abstrakti-

2 Das »Politische« in politischen Konflikten ergibt sich aus dem Bezug auf die Größen »Staat«, »Sicherheit« und »Höchstnormen«: Politische Konflikte weisen stets einen Staatsbezug auf, der sich nicht in einer Staatsbeteiligung äußern muss, sondern der immer dann gegeben ist, wenn der grundlegende Staatszweck, für Sicherheit zu sorgen, betroffen ist. Dies ist der Fall, wenn in einem Territorium eine Verknappung physischer Sicherheit tatsächlich, wahrgenommen oder potenziell vorliegt. Das Potenzial zur Reduzierung von Sicherheit ergibt sich immer dann, wenn ein Konfliktakteur die Verletzung einer Höchstnorm (Verfassungsrecht, Völkerrecht oder Menschenrechte) beklagt und dieser Vorwurf nicht im Rahmen dieser Höchstnorm behandelt wird (bspw. vor einem von dem betreffenden Akteur als legitim erachteten Verfassungsgericht oder internationalen Gerichtshof). 
onsniveau, auf dem wir uns hier insbesondere hinsichtlich der Konfliktgegenstände bewegen, sind die Gemeinsamkeiten der verschiedenen Kommunikationskonstellationen größer als die jeweiligen Unterschiede.

Zum anderen können Konflikttypen anhand der inhaltlichen Bezugspunkte der Konfliktkommunikation in machtpolitische, sozioökonomische sowie kulturelle Konflikte differenziert werden. Wir begreifen Kultur in diesem Zusammenhang als Bedeutungsgewebe, das zur Hervorbringung und Wahrung der Identität eines Kollektivs konstituiert wird (Geertz 1994: 9). Damit gehört alles, was von einer Gesellschaft zur Hervorbringung und Wahrung der kollektiven Identität konstruiert wird, zum Bereich des Kulturellen. Mit der Eingrenzung auf den Identitätsbereich wird ein Kulturbegriff mittlerer Reichweite gewählt. Er grenzt sich ab von dem engen soziologischen Kulturbegriff (Kultur als Komplex aus Standards, Werten und Normen und ihren Symbolisierungen (Hansen 1993; 2000; Mintzel 1993; Parsons/Shils 1990; Schmid 1992)) und von dem weiten ethnologischen Kulturbegriff(Kultur als Inbegriff menschlicher Lebensweise (Held et al. 1999; Huntington 1997; Schwelling 2004)).

Kulturkonflikte sind damit all jene Auseinandersetzungen zwischen Akteuren, in denen die aus eben diesem Bedeutungsgewebe entstehende Identität Thema des Konflikts ist. Hieraus ergeben sich die folgenden Konflikttypen:

\section{Tabelle 1: Konflikttypologie}

\section{Differenzierung nach Konfliktakteuren}

Innerstaatliche Kon- Konflikt zwischen nicht-staatlichen Akteuren innerhalb eines Staates flikte oder zwischen einem Staat und einem nicht-staatlichen Akteur in diesem Staat.

Zwischenstaatliche Konfliktparteien sind hier ausschließlich Staaten.

Konflikte

Transnationale Kon- Konflikt zwischen nicht-staatlichen Akteuren unterschiedlicher natioflikte naler Herkunft oder zwischen einem Staat und nicht-staatlichen Akteuren aus anderen Staaten.

\section{Differenzierung nach Konfliktgegenstand}

Machtpolitische

Konflikte

Hier dreht sich die Konfliktkommunikation um den Zugang zu autoritativen Positionen in Staat und Gesellschaft oder im internationalen System (»Machtverteilung «).

Sozioökonomische Konflikte

Hier bilden die Verteilung von Gütern und Rechten in oder zwischen Gesellschaften sowie die dieser Verteilung zugrundeliegenden Mechanismen den Inhalt der Auseinandersetzung (»ökonomische Teilhabe $\ll)$.

Kulturelle Konflikte Hier bildet Kultur den Inhalt der Kommunikation. 
Politische Konflikte sind in einem strukturellen Kontext verortet. Er bildet den Rahmen der Kommunikation und standardisiert sie, indem er bestimmte Themen und den Einsatz bestimmter Medien zu bestimmten Zeiten durch bestimmte Akteure wahrscheinlicher macht als entsprechend denkbare Alternativen (Krallmann/Ziemann 2001: 249; Hansen 2000: 39; Billington et al. 1991: 5). Der strukturelle Kontext besteht aus zwei Ebenen: zum einen aus dem ökologisch-anthropologischen Kontext der physikalisch-biologischen Umwelt des Menschen (Goffman 1977: 31-32; Parsons 1978; Esser 1999: 92-93) und zum anderen aus dem sozio-kulturellen Kontext. Letzterer zerfällt seinerseits wieder in einen gesellschaftlichen Rahmen (z.B. politische Institutionen, wirtschaftliche und demografischen Strukturen) sowie in einen kulturellen Kontext (d.h.: Kultur im Sinne unserer Definition). Für die Beschäftigung mit kulturellen Konflikten ist in erster Linie dieser kulturelle (Teil-)Kontext von Bedeutung. Er steht mit dem Konfliktgeschehen insofern in einer doppelten Verbindung, als dass er einerseits die kulturellen Strukturen enthält, die konfliktursächlich sein können und die somit Gegenstand der ersten Fragestellung sind. Zum anderen wird der kulturelle Kontext in kulturellen Konflikten selbst zum Thema und damit Gegenstand der zweiten Fragestellung. Letzteres bedarf der zusätzlichen Erläuterung.

Jeder politische Konflikt nimmt als Kommunikation Bezug auf seinen Kontext. Kulturelle Konflikte stechen in dieser Hinsicht allerdings durch eine Besonderheit hervor: Sie nehmen nicht einfach Bezug (Referenz) auf den kulturellen Kontext - in kulturellen Konflikten wird der kulturelle Kontext selbst zum Gegenstand des Konflikts (Introferenz). In herkömmlichen Konflikten bezieht sich die Kommunikation auf einen thematischen Konfliktgegenstand. Im Regelfall wird er in expliziten Forderungen als eindeutig abgrenzbares, interessengeleitetes Konfliktgut formuliert. Damit erscheint der Konfliktgegenstand im Kern verhandelbar. Im Unterschied dazu geht es in kulturellen Konflikten nicht (nur) um Interessen, sondern vor allem um Identität. Kulturelle Konflikte sind Identitätskonflikte und somit schwer zu verhandeln. Auch wenn herkömmliche Konfliktgüter fast immer eine zusätzliche Rolle spielen, konzentriert sich die Kommunikation in einem kulturellen Konflikt doch auf »Konfliktfelder«, d.h. auf ein oder mehrere nicht explizit formulierte, identitätsbezogene Themen. Zu beachten ist, dass Konfliktfelder nicht Motive, sondern Themen darstellen: Sie drücken aus, worum es in dem Konflikt geht, worüber kommuniziert wird und nicht, warum der Konflikt geführt wird, was also seine Ursachen sind (Seul 1999: 564).

Als Konfliktfelder (vgl. Tabelle 2) kommen drei Dimensionen von Kultur in Betracht: Religion, Sprache und geschichtliche Zusammenhänge (nachfolgend als »Historizität« bezeichnet). Mit Neil Smelser (1992: 11), Klaus Hansen (2000: 47) und Niklas Luhmann (1984: 224; 1985: 46-47) lässt sich argumentieren, dass die Hervorbringung und Wahrung der Identität eines Kollektivs mit drei Erfordernissen verknüpft ist: (1) Die Komplexität von Welt und Gesellschaft muss zur Ermöglichung von Identität reduziert werden, (2) die Identität muss zur Verbreitung im Kollektiv kommuniziert werden und (3) die Identität muss zur Erhaltung fortlaufend reproduziert werden. Diese Erfordernisse werden vorrangig durch die Phänomene Religion (Komplexitätsreduktion), Sprache (Kommunikation) und Historizität (Reproduktion) 
erfüllt: (1) Religion ist der Mechanismus zur Komplexitätsreduktion par excellence, da sie die Kontingenz der Welt, also die Unvorhersehbarkeit des Möglichen, weitestmöglich, selbst in postmortaler und transzendentaler Hinsicht, reduziert und dadurch Kontrolle und Kontinuität der Welt und damit individuelle und kollektive Identität ermöglicht. (2) Sprache wiederum ist der Kommunikationsmechanismus par excellence - der Mensch ist ein Sprachwesen, das in seiner Wahrnehmung der Welt und damit der Konstruktion seiner eigenen Identität und der Identität des zugeordneten Kollektivs von der jeweiligen Sprache geprägt wird. (3) Historizität als Geschichtlichkeit im Sinne von Geschichtserfahrung (markante historische Ereignisse) bzw. als narrative Geschichte (historische oder historisierende Herkunftsgeschichte) dient der fortlaufenden Reproduktion des betreffenden Kollektivs durch Tradierung seiner Identität.

Die vorliegende Untersuchung definiert daher kulturelle Konflikte als solche innerstaatlichen, zwischenstaatlichen oder transnationalen politischen Konflikte, in denen die beteiligten Akteure die Konfliktfelder Sprache, Religion (vgl. Fox/Sandler 2006) und/oder Historizität thematisieren. Hervorzuheben ist dabei nochmals, dass kulturelle Konflikte über das Thema des Konflikts bestimmt werden und nicht, wie sonst oft üblich, über die ihnen zugrundeliegenden Ursachen im Sinne von Wirkfaktoren. Kulturelle Konflikte sind nicht notwendigerweise Konflikte, in denen kulturelle Unterschiede zwischen gesellschaftlichen Gruppen (etwa Sprache oder Religion) zum Auftreten eines Konflikts führen. Die Konzeptualisierung kultureller Konflikte und die Ausdifferenzierung von Kultur als soziales Phänomen entlang der drei Dimensionen von Sprache, Religion und Historizität ermöglichen nicht nur die Abgrenzung kultureller Konflikte von anderen Konfliktformen nicht-kultureller Art, sondern auch eine Binnendifferenzierung kultureller Konflikte in unterschiedliche Typen (vgl. Tabelle 2). ${ }^{3}$

3 Diese Definition kultureller Konflikte deckt sich nicht mit in der Literatur gängigen Konzepten wie »ethnische« oder »religiöse« Konflikte. »Ethnische Konflikte« sind politische Konflikte zwischen Ethnien oder zumindest unter Beteiligung mindestens einer Ethnie. Das definierende Merkmal des Konzepts ethnischer Konflikte sind die ethnischen Akteure. Wer die Akteure sind - ethnische Gruppen sind kulturelle Entitäten -, determiniert jedoch nicht, worüber sie kommunizieren: »Ethnische« Konflikte müssen sich nicht zwingend um Kulturelles drehen, sondern können auch um machtpolitische oder sozioökonomische Konfliktgüter geführt werden. Umgekehrt ist selbstverständlich auch nicht jeder kulturelle Konflikt ein »ethnischer « Konflikt. Eine weitere Problematik ist Folgendes: »Ethnien« sind in der Ethnologie oftmals »kleinere« Volksgruppen (z.B. »die Hmong « in Laos), bei denen ein konzertiertes Handeln vorstellbar ist. Anders verhält es sich jedoch bei Religionen (oder auch bei den »Rassen « der angelsächsischen Literatur): »Die Muslime« als Religionsgemeinschaft können nicht als handelnder Akteur konzipiert werden. Daher wird bei Religionen häufig ein Ersatzakteur substituiert (z.B. eine muslimische Bruderschaft in Nigeria). Die Feststellung jedoch, dass eine Organisation mehrheitlich aus Angehörigen einer bestimmten Religion besteht, reicht zur Einstufung des Konflikts als religiös nicht aus. So kann ein Konflikt zwischen zwei Gruppen bestehen, deren Mitglieder v.a. Christen sind, doch damit ist der Konflikt noch nicht zwingend inter-konfessionell (auch wenn bspw. die meisten Mitglieder der CDU und der SPD in Deutschland Christen sind, bilden religiöse Themen in der Kommunikation zwischen den beiden Akteuren nur selten ein Thema). 


\begin{tabular}{|c|c|c|}
\hline Konfliktfeld & Indikator & Beispiel \\
\hline Religion & $\begin{array}{l}\text { Verbaler oder aktiver Verweis auf ein } \\
\text { religiöses Symbol (Person oder Ge- } \\
\text { genstand), der als Thematisierung von } \\
\text { Religion verstanden wird. }\end{array}$ & $\begin{array}{l}\text { Umstrittener Besuch eines Tempels } \\
\text { durch einen Regierungschef oder At- } \\
\text { tentat auf einen religiösen Führer. }\end{array}$ \\
\hline Sprache & $\begin{array}{l}\text { Verbaler oder aktiver Verweis auf ein } \\
\text { sprachliches Symbol (Person oder Ge- } \\
\text { genstand), der als Thematisierung von } \\
\text { Sprache verstanden wird. }\end{array}$ & $\begin{array}{l}\text { Verbot einer Sprache an den Univer- } \\
\text { sitäten oder Erhebung eines Dialekts } \\
\text { zur Sprache. }\end{array}$ \\
\hline Historizität ${ }^{1}$ & $\begin{array}{l}\text { Verbaler oder aktiver Verweis auf ein } \\
\text { Symbol (Person oder Gegenstand) mit } \\
\text { Bezug zu markanten geschichtlichen } \\
\text { Ereignissen oder zur historischen/his- } \\
\text { torisierenden Herkunftsgeschichte, } \\
\text { wobei dieser Verweis als Thematisie- } \\
\text { rung von Historizität verstanden wird. }\end{array}$ & $\begin{array}{l}\text { Umstrittene Errichtung eines Krie- } \\
\text { gerdenkmals oder öffentlicher Dis- } \\
\text { kurs über vorkoloniale Staatserfah- } \\
\text { rung. }\end{array}$ \\
\hline
\end{tabular}

Anmerkungen: 1 = Der hier verwendete Historizitätsbegriff unterscheidet sich von dem der Geschichtswissenschaft, die darunter die Faktizität historischen Geschehens versteht. Innerhalb dieses Konfliktfelds hat auch die Problematisierung der Hautfarbe und Physiognomie ihren Platz - also das, was im angelsächsischen Raum als »Rassenzugehörigkeit« diskutiert wird. Die Hautfarbe »eignet sich aufgrund des langsamen Schwindens der Distinktion besonders zur symbolischen Vergegenwärtigung von Herkunftsgeschichtlichkeit.

Das Design der vorliegenden Studie erfordert drei Formulierungen von Erwartungen über den Effekt kultureller Wirkungsfaktoren auf Konflikte im Allgemeinen und im Hinblick auf die Ursachen kulturell konnotierter Konflikte im Speziellen. Dies geschieht in den folgenden zwei Abschnitten. Innerhalb der Abschnitte wird getrennt auf inner- und zwischenstaatliche Konflikte eingegangen.

Im Hinblick auf die von Kultur ausgehenden Kausaleffekte auf das Konfliktgeschehen werden kulturelle Strukturmuster einer Gesellschaft im Wesentlichen in deren kultureller Fraktionalisierung erfasst, beziehungsweise in ihren Unterformen, der religiösen und der sprachlichen Fraktionalisierung. Das kulturelle Strukturmuster formt die Gesellschaft und die Kommunikationen, aus denen sie besteht. Gesellschaftliche Einheiten sind nur dann als Akteure zu verstehen, wenn und solange sie an dieser Kommunikation beteiligt sind. Dies impliziert gleichzeitig, dass das kulturelle Strukturmuster einer Gesellschaft bestimmte Kommunikationen und damit auch das Auftreten bestimmter Akteure wahrscheinlicher oder unwahrscheinlicher macht. Alternativ ließe sich auch formulieren: Viele Akteure sind nur innerhalb einer Gesellschaft denkbar, die eine ganz bestimmte kulturelle Strukturierung aufweist.

Wenn aber die kulturelle Strukturierung einer Gesellschaft ihre Kommunikationen formt, dann bedeutet dies, dass kulturelle Diversität eine Diversifizierung der Kommunikation nach sich zieht und über Differenzen in der kollektiven Identität kom- 
muniziert wird. Diversifizierung bedeutet also auch steigende Kommunikationsaktivität und eine Zunahme von Akteuren mit untereinander bestehenden Differenzen. Es ist somit zu erwarten, dass eine Zunahme der kulturellen Fraktionalisierung das Auftreten innerstaatlicher Konflikte begünstigt.

Damit lassen sich drei Hypothesen für innerstaatliche Auseinandersetzungen formulieren, die gleich gerichtet sind:

- Die Wahrscheinlichkeit innerstaatlicher Konflikte ist umso größer, je größer (Hypothese 1i) die sprachliche, (Hypothese 2i) die religiöse oder (Hypothese 3i) die kulturelle Fraktionalisierung eines Landes ist.

Zwar ließe sich auch argumentieren, dass eine hohe Fraktionalisierung als »Hyperdiversifikation « von Kommunikation einen dämpfenden Einfluss haben kann. Da die abhängige Variable Kulturkonflikte für diese Untersuchung aber neu bestimmt wurde und der CONIS-Ansatz potenziell ein breiteres und damit anderes Spektrum von Konflikten erwarten lässt als bisher verbreitete Datensätze, sollen diese Überlegungen für die erste Analyse noch zurückgestellt werden. Sie werden weiter unten ausdrücklich wieder aufgegriffen. Da sich die Konfliktgegenstände zwischenstaatlicher Auseinandersetzungen von denen innerstaatlicher Konflikte unterscheiden (Schwank 2010) vermuten wir a priori - außer für die offensichtliche Verbindung zwischenstaatlicher Kulturkonflikte - keinen Effekt kultureller Fraktionalisierung auf die zwischenstaatliche Konfliktbelastung eines Landes. Insofern lassen sich unsere Hypothesen für zwischenstaatliche Auseinandersetzungen wie folgt formulieren:

- Die Wahrscheinlichkeit zwischenstaatlicher Konflikte ist unabhängig von (Hypothese 1z) der sprachlichen, (Hypothese 2z) der religiösen und (Hypothese 3z) der kulturellen Fraktionalisierung eines Landes.

Die zweite, spezifische Forschungsfrage macht es nötig, über den Effekt des kulturellen Rahmens hinaus auch weitere Wirkungshypothesen aufzustellen, die potenzielle nicht-kulturelle Ursachen von (kulturellen) Konflikten erfassen. Im Anschluss an die Ergebnisse der empirischen Konfliktforschung wird dazu ein Grundmodell formuliert, das neben dem kulturellen Kontext weitere Faktoren zur Erklärung der Konfliktwahrscheinlichkeit in einem Land enthält, die sich in der bisherigen empirischen Konfliktforschung als erklärungskräftig erwiesen haben. Das Grundmodell orientiert sich dabei an vergleichbaren Studien in der Forschungsliteratur (Fearon/Laitin 2003; Collier/Hoeffler 2004; Hegre/Sambanis 2006) und beinhaltet Kontrollvariablen für den Entwicklungs- und Demokratisierungsgrad eines Landes sowie Variablen zur außenwirtschaftlichen Verflechtung, kultivierbaren Landfläche, zur Migration sowie zum Wirtschaftswachstum. Erweitert wird dieses Grundmodell zudem um den Faktor youth bulge ${ }^{4}$ der eine starke Herausforderung eines kulturellen Erklärungsansatzes (Huntington 1997) darstellt. Die theoretisch erwarteten Einflüsse der Kontrollvariablen lassen sich wie folgt formulieren:

Analog zu den vorherigen Überlegungen zur kulturellen Fraktionalisierung ist zu erwarten, dass größere Länder heterogener sind als kleinere, was der Argumentation

4 Wagschal et al. (2008); Urdal (2004; 2006); Heinsohn (2003); Fuller (1995; 2004); Fuller/ Pitts (1990). 
Paul Colliers und Anke Hoefflers (2004: 588; 1998: 564) sowie Henrik Urdals (2004: 8) zufolge separatistische Tendenzen und damit Konflikte fördern dürfte. Daraus folgen die Hypothesen,

- dass die Wahrscheinlichkeit innerstaatlicher (Hypothese 4i) und zwischenstaatlicher (Hypothese 4z) Konflikte umso höher ist, je größer die Bevölkerung ist. Wegen der großen Streuung der Werte wurden die herangezogenen Bevölkerungsdaten logarithmiert.

Ein hohes Entwicklungsniveau wird oft mit einem geringeren Risiko innerstaatlicher Konflikte in Verbindung gebracht (z.B. Collier/Hoeffler 2004: 574). Daher erwarten wir,

- dass die Wahrscheinlichkeit innerstaatlicher Konflikte umso höher ist, je geringer das Entwicklungsniveau eines Landes ist (Hypothese 5i). Zwischen Staaten sollte diese Beziehung indes nicht gelten, weshalb wir keinen Zusammenhang erwarten (Hypothese 5z). Der Wohlstand bzw. das Entwicklungsniveau einer Gesellschaft wird von uns durch die Säuglingssterblichkeit abgebildet (ausführlich zu diesem Indikator Sen 1998).

Einem ökonomischen Knappheitsargument entspringt auch die Wahl der dritten Kontrollvariable »kultivierbare Landfläche«, gemessen in Hektar pro Person. Je weniger Landfläche zur Verfügung steht, desto eher dürfte - vor allem bei wachsender Bevölkerung - ein Konflikt um diese knappe Ressource ausbrechen, womit auch die Konfliktwahrscheinlichkeit ansteigen sollte. Daher erwarten wir,

- dass die Wahrscheinlichkeit innerstaatlicher (Hypothese 6i) und zwischenstaatlicher (Hypothese 6z) Konflikte umso höher ist, je geringer die kultivierbare Landfläche pro Person ist.

- Ausgehend von der Überlegung, dass ein Zuwachs an Wohlstand Verteilungskonflikte tendenziell entschärfen sollte, ist zudem zu erwarten, dass das Wachstum des Bruttoinlandsprodukts pro Kopf sowohl das Risiko innerstaatlicher (Hypothese 7i) als auch das zwischenstaatlicher (Hypothese 7z) Konflikte senkt. $^{5}$

Die These vom liberalen Handelsfrieden (Rosecrance 1986; Polachek 1997; McDonald 2004; Oneal/Russett 1999) geht auf Basis einer positiven Bewertung von Freihandel davon aus, dass stärker in den internationalen Handel eingebundene Länder friedlicher sind. Im Sinne dieser These erwarten wir daher,

- dass eine hohe Weltmarkintegration (gemessen als Export von Gütern und Dienstleistungen in Prozent des Bruttoinlandsprodukts) einen dämpfenden Effekt auf die zwischenstaatliche Konfliktwahrscheinlichkeit hat (Hypothese 8z).

Für innerstaatliche Konflikte ist das Bild weniger einheitlich.

5 Alternativ ließe sich argumentieren, statt der Wachstumsrate selbst deren Veränderung über Zeit als Indikator heranzuziehen, um so mehr auf die vom Individuum wahrgenommenen Änderungen der wirtschaftlichen Lage abzustellen. Zwar ist es zweifellos korrekt, dass Individuen erkennen, ob sich die ökonomische Lage schneller oder langsamer ändert als in der Vergangenheit. Für das von uns angeführte absolute Knappheitsargument dürfte die Wirkung eines allgemeinen wirtschaftlichen Auf- oder Abwärtstrends aber von größerer Bedeutung sein. 
Wie Katherine Barbieri und Rafael Reuveny (2005) ausführen, stehen sich hier neben Stimmen, die keine Beziehung zwischen Handel und Konfliktrisiko sehen zwei gegensätzliche Positionen gegenüber: Eine Seite verweist auf nivellierende und wohlstandssteigernde Effekte von Handel, auf dessen Fähigkeit, staatliche Strukturen zu stärken oder auf das alle potenziellen Konfliktparteien disziplinierende Risiko, im Fall einer Auseinandersetzung keine Handelsgewinne mehr zu erhalten. Die andere Seite hingegen wendet unter anderem ein, dass derartige Gewinne meist nur kleinen Eliten und nicht breiten Bevölkerungsschichten zuteilwerden, was Ungleichheit und damit Unzufriedenheit fördert. Als eine davon unabhängige Überlegung verfolgt die Political Instability Task Force (Goldstone et al. 2000: 10-11) zudem die Überlegung, dass ein geringer Außenhandel Indiz für grundlegende strukturelle Probleme sein könnte. Das Handelsniveau mancher Länder wäre demzufolge gegenüber einem - in Abhängigkeit von der Ländergröße variierenden - »natürlichen « Handelsniveau reduziert, beispielsweise durch rent-seeking lokaler Eliten, Korruption oder durch unwirksame juristische Strukturen, die der eigentliche Grund für eine negative Beziehung zwischen Handelsniveau und Konfliktrisiko seien. Wir gehen davon aus,

- dass die Verbindung zwischen Handel und Konfliktrisiko auf innerstaatlicher Ebene dem zwischenstaatlichen Muster folgt und erwarten, dass eine hohe Weltmarktintegration das Risiko innerstaatlicher Konflikte senkt (Hypothese 8i).

Im Anschluss an Immanuel Kant - Staaten mit demokratischer Verfassung bekämpfen einander nicht -, wird der Demokratisierungsgrad meist als weitere entscheidende Größe für das Konfliktrisiko eines Landes angeführt. Während oft davon ausgegangen wird, dass das zwischenstaatliche Konfliktrisiko mit dem Anstieg des Demokratieniveaus sinkt, ist innerstaatlich eine solche als »domestic democratic peace» (Henderson/Singer 2000: 279, Fn. 3) bezeichnete Verbindung theoretisch allerdings nicht eindeutig. Zwar können in Autokratien Konflikte nicht offen ausgetragen werden, was ihr Konfliktpotenzial eigentlich erhöhen sollte, umgekehrt kann Protest in diesen Systemen aber meist auch effektiver unterdrückt werden (vgl. z.B. Urdal 2006: 613; Fearon/Laitin 2003: 79; Henderson/Singer 2000: 279). Demokratien wiederum haben hingegen die Möglichkeit, Konflikte gewaltfrei zu bearbeiten. Aus diesen Gründen ist wiederholt davon ausgegangen worden, dass die Verbindung von Demokratie und innenpolitischer Gewalt nichtlinear sein könne (Gurr 1968; 1970; Muller/Weede 1990: 626; Hegre et al. 2001), vor allem weil zwischen beiden Polen stehende Systeme wegen ihres uneinheitlichen Institutionensystems zu keiner der beiden Lösungen in der Lage seien (Henderson/Singer 2000: 279-280; Gleditsch 1995). Allerdings wird auch argumentiert, dass halbdemokratische Systeme in erster Linie Systeme in einem akuten Transformationsprozess seien, der enttäuschten Gruppen Rebellion attraktiv erscheinen ließe (Hegre et al. 2001: 34; Henderson/Singer 2000: 279). In der jüngsten Literatur wird die sonst meist als relativ stabil charakterisierte Beziehung (Hegre/ Sambanis 2006: 526) kritisch gesehen (Strand 2007; Fjelde 2008), vor allem wegen potenzieller Messprobleme.

Für unsere Analyse formulieren wir im Einklang mit der herrschenden Meinung gleichwohl die Erwartung, 
- dass Staaten mit einem mittleren Demokratisierungsniveau ein höheres Risiko innerstaatlicher Konflikte haben als Autokratien und Demokratien (Hypothese $9 i$ ). Für zwischenstaatliche Konflikte erwarten wir, dass das Risiko einer Auseinandersetzung mit steigender Demokratisierung zurückgeht (Hypothese 9z).

Als weitere Einflussgröße wird die Migration über die Grenzen eines Landes hinweg herangezogen. Sie kann unterschiedliche Ursachen haben, wie z.B. ökonomische Attraktivität (für Immigration) oder aber auch Konflikte (für Emigration), weshalb ein massives Endogenitätsproblem bei dieser Variable vorliegt. Im Folgenden wird davon ausgegangen,

- dass Emigration die Konfliktwahrscheinlichkeit in einem Land senkt, während Immigration diese erhöht (Hypothese 10i).

- Für zwischenstaatliche Konflikte ist die Kausalität aufgrund des Endogenitätsproblems uneindeutiger. Da Länder mit Emigration zumeist auch von hoher Violenz geprägt sind, erwarten wir hier ein positives Vorzeichen (Hypothese 10z).

Als letzte Variable des Grundmodells wird auf youth bulges (Heinsohn 2003; Urdal 2006; Wagschal et al. 2008) kontrolliert, die - bedenkt man, dass Huntington (1997: 433) ihnen eine zentrale Rolle als Motor im »Kampf der Kulturen« zuweist - auch als Herausforderung an eine kulturell motivierte Erklärung verstanden werden können. Ausgangspunkt der These ist die Erkenntnis, dass der Großteil der Protagonisten politischer, aber auch anderer Formen von Gewalt, jung und männlich ist (Mesquida/ Wiener 1999; Goldstone 1991: 136-137; Urdal 2004: 3). Der Überlegung zufolge kann ein großer Bevölkerungsanteil nach Status und Anerkennung strebender junger Männer als youth bulge (von engl. bulge = Wölbung, Beule) das Risiko inner- und zwischenstaatlicher Konflikte erhöhen, vor allem dann, wenn den Statusbestrebungen keine adäquaten Aufstiegsmöglichkeiten gegenüberstehen. Die Effekte eines solchen Jugendüberschusses - hier erfasst als der Anteil junger Männer zwischen 15 und 24 an der männlichen Bevölkerung über 14 - können durch Faktoren wie z.B. Bevölkerungsdichte verstärkt, aber auch beispielsweise durch ökonomisches Wachstum, Migration oder Krankheiten wie Aids abgeschwächt werden (vgl. Wagschal et al. 2008). Für unsere Analyse erwarten wir,

- dass die Wahrscheinlichkeit innerstaatlicher (Hypothese 11i) und zwischenstaatlicher (Hypothese 11z) Auseinandersetzungen umso höher ist, je größer der Anteil junger Männer an der männlichen Bevölkerung eines Landes ist.

\section{Daten und Methode}

Die Zuordnung empirischer Konflikte zu einem oder mehreren kulturellen Konfliktfeldern erfolgt auf Grundlage des an der Universität Heidelberg entwickelten CONISDatensatzes. CONIS wertet ausschließlich Informationen aus öffentlich zugänglichen Nachrichtenquellen qualitativ aus und bereitet sie zum Zwecke einer Ereignisdatenanalyse auf. CONIS unterscheidet sich von anderen Datenbanken wie dem Correlates of War-Projekt (COW (Singer/Small 1972; Small/Singer 1982)) und dem Uppsala Conflict Data Program (UCDP 2010 (Gleditsch et al. 2002)) durch drei wesentliche 
Merkmale: (1) CONIS erfasst alle Formen politischer Konflikte weltweit seit 1945 und beschränkt sich nicht auf einzelne Regionen oder auf gewaltsame Konflikte. (2) CONIS erfasst Konfliktdynamiken, also die individuellen Entwicklungsphasen der einzelnen Konflikte. (3) CONIS ist eine quantitative Datenbank auf qualitativer Grundlage: Die Messung und Kategorisierung der Konflikte basiert nicht wie bei anderen Forschungsansätzen auf der Zahl kampfbedingter Todesopfer sondern auf einer Inhaltsanalyse der zwischen den Akteuren stattfindenden Kommunikationen und Handlungen. Als Ergebnis der methodischen Erneuerung erfasst CONIS eine wesentlich höhere Anzahl politischer Konflikte. Dies gilt insbesondere für innerstaatliche Konflikte, für die oftmals keine oder nur sehr ungenaue Todesopferangaben vorliegen. Außerdem erzwingt die Untersuchung von Konfliktdynamiken die Aufteilung des Konfliktgeschehens in verschiedene Teilkonflikte, die in anderen Datensätzen als ein größerer Konflikt codiert werden. So umfasst der CONIS Datensatz zum hier verwendeten Stand (2007) insgesamt 761 Konflikte und 13.126 Jahresintensitätswerte. Der am häufigsten zitierte Datensatz des Correlates of War-Projektes (Sarkees 2000) stellt für den gleichen Zeitraum lediglich 144 Kriegsdatensätze ohne Jahresdaten zur Verfügung. Der ebenfalls weit verbreitete Datensatz des Uppsala Conflict Data Program (UCDP 2010 Armed Conflict Dataset v. 4-2008, 1946-2007) enthält 235 bewaffnete Grundkonflikte und 1.912 Jahresdaten. Da der CONIS Datensatz somit eine mehrfache Datenmenge im Vergleich zu anderen Datensätzen enthält, liegt es nahe, dass durch die Analyse der CONIS Daten Ergebnisse erzielt werden, die von bisherigen Untersuchungen abweichen (vgl. Schwank 2010).

Das für CONIS entwickelte dynamische Konfliktmodell umfasst insgesamt fünf Intensitätsstufen. Die erste Stufe (»Disput«) markiert die Artikulation eines Interessengegensatzes, die zweite (»gewaltlose Krise «) die Drohung mit Gewalt. Die dritte Stufe (»gewaltsame Krise«) beinhaltet die punktuelle, begrenzte Anwendung von Gewalt, in der vierten (»begrenzter Krieg«) wird Gewalt geplant eingesetzt, ohne aber das Ziel zu verfolgen, den Gegner vollständig niederzuwerfen. Es geht eher darum, ihn durch massive Gewalt zum Einlenken zu bewegen. Die fünfte Stufe (»Krieg«) schließlich ist die systematische Anwendung von Gewalt mit dem Ziel, den Gegner niederzuwerfen und ihm den eigenen Willen aufzuzwingen (Schwank 2010; Wagschal et al. 2008). Das weltweite Konfliktpanorama wird von CONIS in inner- und zwischenstaatliche Konflikte untergliedert, so dass ein geeigneter Datenbestand vorliegt, um die oben formulierten Hypothesen zu prüfen.

Neben der Zuordnung der verschiedenen Phasen eines Konflikts ist hier auch die Typologisierung eines Konflikts anhand seiner Themen von Bedeutung. Die in Tabelle 1 vorgenommene Differenzierung können wir zu diesem Zweck leicht vereinfachen: Die machtpolitischen und sozioökonomischen Konflikte fassen wir zur Kategorie der »nicht-kulturellen Konflikte« zusammen. Die Klassifikation eines Konflikts als »kultureller Konflikt« beruht auf der Beobachtung, ob in der Konfliktkommunikation kulturelle Gegenstände thematisiert werden, hängt also davon ab, auf welche Themen mindestens einer der beteiligten Konfliktakteure (z.B. eine Regierung) oder ein ihm zugeordneter Akteur (bspw. eine Armeeführung) Bezug nimmt. $\mathrm{Ob}$ und in welcher Form eine solche Bezugnahme vorliegt, beruht auf den kommu- 
nizierten Intentionen dieses Akteurs und auf dem gleichfalls kommunizierten Verständnis des oder der Adressaten, also auf Kommunikationen, wie sie in öffentlich zugänglichen Quellen erkennbar werden. Ob ein Konfliktgegenstand als kulturell definiert wird, hängt davon ab, ob die Akteure eines der oben genannten Konfliktfelder »aktivieren«. Zur Operationalisierung der Aktivierung der kulturellen Konfliktfelder wird als Indikator jeweils der verbale oder aktive Verweis auf ein entsprechendes Symbol (Person oder Gegenstand) durch einen Konfliktakteur herangezogen, der vom Gegenüber als Thematisierung von Religion, Sprache oder Geschichtlichkeit (im Sinne geschichtlicher Ereignisse oder der Herkunftsgeschichte) verstanden wird.

Die nachfolgende Tabelle 3 gibt Aufschluss über die Anzahl und die Verteilung der in der CONIS-Datenbank (Stand: Dezember 2007) registrierten Konflikte. Erfasst wird die Anzahl der Grundkonflikte nach der höchsten erreichten Konfliktstufe, unabhängig von ihrer Dauer.

Tabelle 3: Anzahl und Anteil der inner- bzw. zwischenstaatlichen kulturellen und nicht-kulturellen Konflikte nach der jeweils höchsten erreichten Konfliktintensität (1945-2007)

\begin{tabular}{|c|c|c|c|c|c|c|}
\hline \multicolumn{7}{|c|}{ Innerstaatliche Konflikte } \\
\hline Intensitätsstufe: & $\begin{array}{l}\text { Disput } \\
\text { (1) }\end{array}$ & $\begin{array}{l}\text { gewalt- } \\
\text { lose } \\
\text { Krise } \\
(2)\end{array}$ & $\begin{array}{l}\text { gewalt- } \\
\text { same } \\
\text { Krise } \\
(3)\end{array}$ & $\begin{array}{l}\text { be- } \\
\text { grenzter } \\
\text { Krieg } \\
(4)\end{array}$ & $\begin{array}{c}\text { Krieg } \\
(5)\end{array}$ & (Summe) \\
\hline $\begin{array}{ll}\text { 兰 } & \text { nicht-kulturelle } \\
\text { Konflikte } \\
\text { (Anteil in Prozent) }\end{array}$ & $\begin{array}{c}2 \\
(0,95)\end{array}$ & $\begin{array}{c}27 \\
(12,86)\end{array}$ & $\begin{array}{c}90 \\
(42,86)\end{array}$ & $\begin{array}{c}57 \\
(27,14)\end{array}$ & $\begin{array}{c}34 \\
(16,19)\end{array}$ & $\begin{array}{l}210 \\
(100)\end{array}$ \\
\hline $\begin{array}{ll}\text { Eี kulturelle Konflikte } \\
\text { (Anteil in Prozent) }\end{array}$ & $\begin{array}{c}10 \\
(3,68)\end{array}$ & $\begin{array}{c}31 \\
(11,40)\end{array}$ & $\begin{array}{c}85 \\
(31,25)\end{array}$ & $\begin{array}{c}97 \\
(35,66)\end{array}$ & $\begin{array}{c}49 \\
(18,01)\end{array}$ & $\begin{array}{c}272 \\
(100)\end{array}$ \\
\hline \multicolumn{7}{|c|}{ Zwischenstaatliche Konflikte } \\
\hline Intensitätsstufe: & $\begin{array}{l}\text { Disput } \\
\text { (1) }\end{array}$ & $\begin{array}{l}\text { gewalt- } \\
\text { lose } \\
\text { Krise } \\
(2)\end{array}$ & $\begin{array}{l}\text { gewalt- } \\
\text { same } \\
\text { Krise } \\
(3)\end{array}$ & $\begin{array}{l}\text { be- } \\
\text { grenzter } \\
\text { Krieg } \\
(4)\end{array}$ & $\begin{array}{c}\text { Krieg } \\
(5)\end{array}$ & (Summe) \\
\hline \multirow{2}{*}{ 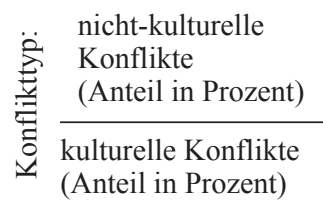 } & $\begin{array}{c}43 \\
(19,82)\end{array}$ & $\begin{array}{c}89 \\
(41,01)\end{array}$ & $\begin{array}{c}55 \\
(25,35)\end{array}$ & $\begin{array}{c}18 \\
(8,29)\end{array}$ & $\begin{array}{c}12 \\
(5,53)\end{array}$ & $\begin{array}{c}217 \\
(100)\end{array}$ \\
\hline & $\begin{array}{c}5 \\
(8,06)\end{array}$ & $\begin{array}{c}20 \\
(32,26)\end{array}$ & $\begin{array}{c}12 \\
(19,35)\end{array}$ & $\begin{array}{c}7 \\
(11,29)\end{array}$ & $\begin{array}{c}18 \\
(29,03)\end{array}$ & $\begin{array}{c}62 \\
(100)\end{array}$ \\
\hline
\end{tabular}

Entsprechend der Trennung zwischen Konfliktgütern und Konfliktfeldern wird auf eine graduelle Unterscheidung zwischen starker und schwacher Thematisierung des 
kulturellen Kontexts verzichtet. Stattdessen werden die einzelnen politischen Konflikte über eine dichotome Differenzierung den kulturellen bzw. den nicht-kulturellen Konflikten zugeordnet. Die eindeutige Abgrenzung dieser Konfliktgruppe basiert auf zwei Bestimmungsmerkmalen: (1) Die kulturelle Thematisierung des Konflikts kann nur über die Entscheidungsträger einer Konfliktpartei erfolgen und (2) aus den Kommunikationen der Konfliktparteien kann konkludent geschlossen werden, dass die Thematisierung des kulturellen Konflikts von den Konfliktparteien als solche verstanden wurde. ${ }^{6}$ Damit wurde sich bewusst gegen eine einfache Zählregel entschieden, welche die Klassifikation eines Konflikts als kulturell/nicht-kulturell ausschließlich aus der Häufigkeit abgeleitet hätte, mit der die Akteure Bezug auf ein Konfliktfeld nehmen. Denn letztlich lässt sich weder allgemein begründen, wie vieler Bezugnahmen es jeweils bedarf, damit ein Konflikt einer bestimmten Konfliktklasse zugeordnet wird, noch lässt die Zahl der Nennungen als solche Rückschlüsse auf die Bedeutung des Konfliktfeldes zu, da Akteure cheap talk betreiben können, d.h. in einer Kommunikation mit Dritten nicht-authentische Deutungen und Begründungen ihres Handelns bieten. Dementsprechend lassen sich auch keine theoretisch begründeten Schwellenwerte angeben, welche die Grenze zwischen bestimmten Konflikttypen markieren. Vielmehr handelt es sich bei der hier gewählten Vorgehensweise um eine kontextualisierende und inhaltsanalytische Interpretation des Datenmaterials. ${ }^{7}$

Hinsichtlich der empirischen Relevanz kultureller Konflikte für das globale Konfliktgeschehen zeigen die Auswertungen in Tabelle 3 zweierlei:

- Kulturelle Konflikte bilden einen signifikanten Anteil am weltweiten Konfliktgeschehen, insbesondere auf innerstaatlicher Ebene. Von den 761 in CONIS erfassten Konflikten sind 44 Prozent Kulturkonflikte. Mit 81 Prozent ist die große Mehrheit der kulturellen Konflikte innerhalb von Staaten zu finden, nur 19 Prozent spielen sich zwischen Staaten ab. Betrachtet man nur den Anteil der Gewaltkonflikte (Stufe 3-5), sind sogar 86 Prozent der kulturellen Konflikte innerstaatlich.

- Deutlich zu erkennen ist außerdem eine höhere Gewaltneigung von kulturellen im Vergleich zu nicht-kulturellen Konflikten: Auf innerstaatlicher Ebene sind 31 Prozent der kulturellen Konflikte zumindest zeitweise mit Gewalt ausgetragen worden (d.h. die höchste gemessene Intensität war Stufe 3), 54 Prozent erreichten sogar die Stufen 4 und 5 (begrenzter Krieg und Krieg). Bei den nicht-kulturellen Konflikten ist dagegen der Anteil der weniger gewaltsamen Konflikte (maximal

6 Die Thematisierung von Kulturellem durch Akteur A und das Verstehen der diesbezüglichen Handlungen und Kommunikation durch Akteur B als Thematisierung von Kulturellem müssen mithin zusammenkommen, um einen kulturellen Konflikt zu konstituieren. So thematisiert bspw. die Tötung buddhistischer Mönche im Süden Thailands durch muslimische Separatisten Kulturelles (hier: Religiöses), und dies wird von der thailändischen Regierung auch so verstanden. Umgekehrt war die Zerstörung von Kirchengebäuden durch die Bombenangriffe auf Deutschland im Zweiten Weltkrieg von den Alliierten nicht als Thematisierung von Kulturellem (d.h. Religiösem) gemeint und wurde von der deutschen Führung auch nicht so verstanden.

7 Diese Interpretation wurde im Laufe des diesem Beitrag zugrundeliegenden Forschungsprozesses von den Autoren durchgeführt. Die dabei generierten Daten sind nun Teil der CONISDatenbank. 
Stufe 3) mit 43 Prozent höher, dafür erreichten nur 43 Prozent ein Gewaltniveau der Stufen 4 oder 5 . Noch deutlicher ist das Muster bei den zwischenstaatlich ausgetragenen Kulturkonflikten: Hier hatten 19 Prozent der registrierten Konflikte die maximale Stufe 3, in 40 Prozent der Fälle wurde zeitweise das Gewaltniveau eines (begrenzten) Krieges (Stufe 4 und 5) gemessen. Im Vergleich dazu erreichten zwar 25 Prozent der nicht-kulturellen Konflikte zwischen Staaten die Intensitätsstufe 3, der Anteil von Konflikten mit einer maximalen Intensität der Stufe 4 oder 5 ist mit 14 Prozent jedoch deutlich geringer.

Für die statistische Auswertung wurden die Konflikte als Länderjahre erfasst, d.h. für jedes Land liegt für jedes Jahr ein eigener CONIS-Wert vor. War ein Land in mehrere Konflikte verwickelt, wurde jeweils das höchste im betreffenden Jahr gemessene Konfliktniveau herangezogen. Um die Auswertung mittels logistischer Regression ${ }^{8}$ zu ermöglichen, wurden die Konfliktwerte dichotomisiert, d.h. jedes Jahr, in dem das Konfliktniveau die Stufe 3, 4 oder 5 erreichte, wurde mit 1 codiert, für die anderen Jahre wurde der Wert 0 vermerkt. Damit wurde eine weite Erfassung des gewaltsamen Konfliktaustrags gewählt, die Konflikte aller Gewaltstufen gleich stark gewichtet.

Die im Grundmodell eingesetzten Makrovariablen stammen aus den Beständen der Vereinten Nationen (Bevölkerung, Säuglingssterblichkeit, Migration und youth bulge (United Nations Population Division 2005)), der Weltbank (Landwirtschaftlich nutzbare Fläche pro Person sowie Export von Gütern und Dienstleistungen (World Bank 2005)) und vom Center for International Comparisons of Production, Income and Prices der Universität Pennsylvania (BIP-Wachstum pro Kopf (Heston et al. 2006)). Wegen der hohen Anzahl verfügbarer Messpunkte wurde für das Demokratisierungsniveau der Polity-IV-Index (Jaggers/Gurr 1995; Marshall/Jaggers 2005) verwendet, der Länder auf einer Skala von -10 für reine Autokratien bis +10 für reine Demokratien einteilt. Um den vermuteten nichtlinearen Zusammenhang zwischen Konfliktrisiko und Demokratie zu erfassen, wurden neben dem regulären Polity- $I V$ Index die Werte zusätzlich quadriert in die Regressionsgleichung eingebracht (vgl. Urban/Mayerl 2006: 207-208). Da zu erwarten steht, dass die Konfliktwahrscheinlichkeit zu beiden Enden der Polity-Skala absinkt, sollte die quadrierte Variable in der Regression ein negatives Vorzeichen besitzen.

Als zentrale unabhängige Faktoren zur Erfassung des kulturellen Kontexts werden die sprachliche, die religiöse sowie die kulturelle Fraktionalisierung eines Landes (letztere als arithmetisches Mittel der beiden anderen Indizes) verwendet. Sowohl die

8 Die binäre logistische Regression (Greene 1993; Liao 1994; Andreß et al. 1997) analysiert Zusammenhänge zwischen metrischen unabhängigen Variablen und einer dichotomen abhängigen Variable $(0,1)$. Anders als bei der linearen Regression betrachtet man dabei aber nicht den konkret erwarteten Wert der abhängigen Variable, sondern vielmehr die Eintrittswahrscheinlichkeit eines Ereignisses, im vorliegenden Fall eines Konfliktes ( $\mathrm{Y}=1)$ oder einer niedrigeren Konfliktstufe $(\mathrm{Y}=0)$. Eine weitere wesentliche Differenz zur linearen Regression ist auch die unterschiedliche Interpretation der Regressionskoeffizienten, da diese in den logistischen Regressionsverfahren nicht so direkt und eindeutig zu lesen sind wie im linearen Modell. Am einfachsten ist es noch, die Ergebnisse als sogenannte odds (Wahrscheinlichkeitsverhältnisse) zu berechnen, was jedoch eine Umrechnung der Koeffizienten des Regressionsoutputs erfordert. 
sprachliche als auch die religiöse Fraktionalisierung wurden berechnet als 1 - Herfindahl-Index auf Basis der jeweiligen Bevölkerungsanteile. ${ }^{9}$ Der Indexwert variiert zwischen 0 und 1, wobei hohe Indexwerte einer starken kulturellen Fraktionalisierung entsprechen, niedrige Werte dagegen homogene Bevölkerungen anzeigen.

\section{Empirische Analyse}

Die vorliegende Studie verbindet zwei Perspektiven. Zum einen wird gefragt, wie das kulturelle setup einer Gesellschaft das Konfliktrisiko beeinflusst. Zum anderen werden die Ursachen von kulturellen Konflikten untersucht. Für die erste Frage ist die abhängige Variable die gesamte Konfliktbelastung eines Landes, für die zweite sind dagegen allein die kulturellen Konflikte relevant. Da wir sowohl inner- als auch zwischenstaatliche Konflikte betrachten, ergeben sich insgesamt vier mögliche Kombinationen, was auch die Gliederung der empirischen Analyse bestimmt: In einem ersten Schritt prüfen wir, inwieweit Kultur als Ursache innerstaatlicher Konflikte wirken kann. Ein zweiter Schritt verengt dann den Fokus auf kulturelle Konflikte und versucht zu klären, wie es zu innerstaatlichen Auseinandersetzungen kommt, in denen Kultur als Thema eine prominente Rolle spielt. Nach der gleichen Vorgehensweise analysieren wir im dritten und vierten Abschnitt zwischenstaatliche Konflikte. Um die Übersichtlichkeit zu erhöhen, sprechen wir bei der abhängigen Variable von »allen Konflikten« bzw. vom »Konfliktpanorama«, wenn die Rolle von Kultur als mögliche Ursache untersucht wird. Steht dagegen Kultur als Thema im Vordergrund, verwenden wir die Bezeichnung »Kulturkonflikte«.

\subsection{Kultur als Ursache innerstaatlicher Konflikte}

In einem ersten Schritt betrachten wir den Einfluss kultureller Faktoren als Ursache von Konflikt. Die Befunde in Tabelle 4 zeichnen ein differenziertes Bild.

9 Quelle für die sprachliche Fraktionalisierung ist der Ethnologue-Datensatz (Gordon 2005). Quelle für die Berechnung der religiösen Fraktionalisierung ist das Jahrbuch 2007 der Encyclopaedia Britannica. 
Tabelle 4: Bestimmungsfaktoren innerstaatlicher Konfliktwahrscheinlichkeit 1950 bis 2005 (alle Konflikte; binäre logistische Regression)

\begin{tabular}{|c|c|c|c|}
\hline & $\begin{array}{c}(1) \\
\text { CONIS }(3,4,5)\end{array}$ & $\begin{array}{c}(2) \\
\text { CONIS }(3,4,5)\end{array}$ & $\begin{array}{c}(3) \\
\text { CONIS }(3,4,5)\end{array}$ \\
\hline Konstante & $\begin{array}{c}-7,909 \\
(0,511)^{* * *}\end{array}$ & $\begin{array}{c}-8,066 \\
(0,514)^{* * *}\end{array}$ & $\begin{array}{c}-8,326 \\
(0,515)^{* * *}\end{array}$ \\
\hline Bevölkerung (logarithmiert) & $\begin{array}{c}0,577 \\
(0,032)^{* * *}\end{array}$ & $\begin{array}{c}0,628 \\
(0,033)^{* * *}\end{array}$ & $\begin{array}{c}0,610 \\
(0,033)^{* * *}\end{array}$ \\
\hline $\begin{array}{l}\text { Säuglingssterblichkeit } \\
\text { (pro } 1000 \text { Geburten, UNWPP) }\end{array}$ & $\begin{array}{c}0,001 \\
(0,001)\end{array}$ & $\begin{array}{c}0,004 \\
(0,001)^{* * *}\end{array}$ & $\begin{array}{c}0,003 \\
(0,001)^{* * *}\end{array}$ \\
\hline $\begin{array}{l}\text { Kultivierbare Landfläche } \\
\text { (Hektar pro Person, WDI) }\end{array}$ & $\begin{array}{c}-0,629 \\
(0,141)^{* * *}\end{array}$ & $\begin{array}{c}-0,576 \\
(0,148)^{* * *}\end{array}$ & $\begin{array}{c}-0,584 \\
(0,141)^{* * *}\end{array}$ \\
\hline $\begin{array}{l}\text { Wachst. BIP/Kopf, int. US\$, PPP, konst. Prei- } \\
\text { se (Penn World Tables) }\end{array}$ & $\begin{array}{c}-0,015 \\
(0,006)^{* *}\end{array}$ & $\begin{array}{c}-0,016 \\
(0,006)^{* * *}\end{array}$ & $\begin{array}{c}-0,017 \\
(0,006)^{* * *}\end{array}$ \\
\hline $\begin{array}{l}\text { Export von Gütern und Dienstleistungen (Pro- } \\
\text { zent des BIP, WDI) }\end{array}$ & $\begin{array}{l}-0,003 \\
(0,003)\end{array}$ & $\begin{array}{c}0,004 \\
(0,003)\end{array}$ & $\begin{array}{c}0,002 \\
(0,003)\end{array}$ \\
\hline $\begin{array}{l}\text { Demokratisierungsgrad } \\
\text { (Polity IV: }-10 \text { bis }+10)\end{array}$ & $\begin{array}{c}0,050 \\
(0,007)^{* * *}\end{array}$ & $\begin{array}{c}0,052 \\
(0,007)^{* * *}\end{array}$ & $\begin{array}{c}0,052 \\
(0,007)^{* * *}\end{array}$ \\
\hline $\begin{array}{l}\text { Demokratisierungsgrad } \\
\text { (Polity IV, quadriert) }\end{array}$ & $\begin{array}{c}-0,008 \\
(0,001)^{* * *}\end{array}$ & $\begin{array}{c}-0,008 \\
(0,001)^{* * *}\end{array}$ & $\begin{array}{c}-0,007 \\
(0,001)^{* * *}\end{array}$ \\
\hline $\begin{array}{l}\text { Nettomigration pro } 1000 \text { Einw. } \\
\text { (positive Werte = Immigration) }\end{array}$ & $\begin{array}{c}-0,014 \\
(0,006)^{* *}\end{array}$ & $\begin{array}{c}-0,012 \\
(0,006)^{*}\end{array}$ & $\begin{array}{l}-0,010 \\
(0,006)\end{array}$ \\
\hline $\begin{array}{l}\text { Youth Bulge: Anteil der 15-24-jährigen Män- } \\
\text { ner an Männern über } 14 \text { Jahre }\end{array}$ & $\begin{array}{c}0,060 \\
(0,008)^{* * *}\end{array}$ & $\begin{array}{c}0,057 \\
(0,009)^{* * *}\end{array}$ & $\begin{array}{c}0,063 \\
(0,009)^{* * *}\end{array}$ \\
\hline Index der sprachlichen Fraktionalisierung & $\begin{array}{c}0,500 \\
(0,137)^{* * *}\end{array}$ & & \\
\hline Index der religiösen Fraktionalisierung & & $\begin{array}{c}-1,453 \\
(0,195)^{* * *}\end{array}$ & \\
\hline Index der kulturellen Fraktionalisierung & & & $\begin{array}{l}-0,249 \\
(0,192) \\
\end{array}$ \\
\hline Pseudo- $\mathrm{R}^{2}$ nach Nagelkerke & 0,242 & 0,256 & 0,241 \\
\hline Einbezogene Fälle (N) & 4427 & 4421 & 4421 \\
\hline
\end{tabular}

Anmerkungen: Die verwendete Methode ist die binäre logistische Regression, die abhängige Variable ist die dichotome Variable zur Erfassung der Konfliktintensität $(0=$ niedrige bzw. keine Konflikte; $1=$ hohe Konfliktintensität). Die Zahlen bei der abhängigen Variable in Klammern geben die aggregierten Konfliktintensitäten der CONIS-Datenbank an. Dargestell sind die Koeffizienten für die logistische Regression (erster Wert) sowie die zugehörigen Standardfehler (in Klammern) mit: * = signifikant auf dem $10 \%$ Niveau, $* *=$ signifikant auf dem $5 \%$-Niveau, *** = signifikant auf dem $1 \%$-Niveau (zweiseitige Fragestellung beim Hypothesentest). 
Für die sprachliche Fraktionalisierung zeigt sich der vermutete positive Zusammenhang zwischen Indexwert und Konfliktrisiko. Demnach nimmt in einem Land das Risiko eines Konflikts mit steigender sprachlicher Fraktionalisierung zu. Für den Index der religiösen Fraktionalisierung gilt dies nicht. Im Widerspruch zu den formulierten Erwartungen haben Länder mit einer höheren religiösen Fraktionalisierung dem Koeffizienten zufolge ein geringeres Konfliktrisiko als homogenere Staaten. Weniger überraschend ist vor dem Hintergrund dieses gemischten Befundes das Ergebnis des dritten Modells: Da der kombinierte Index der kulturellen Fraktionalisierung die gegenläufige Wirkung der religiösen und sprachlichen Fraktionalisierung gleichzeitig erfasst, ist der Koeffizient zwar negativ, aber insignifikant.

Der Blick auf die Kontrollvariablen offenbart einen signifikanten Einfluss der youth bulge-Variablen (dem Anteil junger Männer zwischen 15 und 24) und auch für die meisten anderen Kontrollvariablen zeigt sich das theoretisch erwartete Vorzeichen. Die Koeffizienten sind in fast allen Modellen signifikant. Den Ergebnissen aus Tabelle 4 zufolge sind damit eine große Bevölkerung und teilweise auch Immigration mit einem erhöhten innerstaatlichen Konfliktrisiko korreliert. Auch die Säuglingssterblichkeit als Maßzahl für den Entwicklungsstand einer Gesellschaft ist in zwei der drei Gleichungen hochsignifikant, was auf den vermuteten Zusammenhang zwischen Entwicklungsniveau und Konfliktrisiko hindeutet - je höher die Säuglingssterblichkeit in einem Land ist, desto höher ist auch die Wahrscheinlichkeit eines gewaltsamen Konflikts.

Länder mit einer größeren Verfügbarkeit von Ackerland pro Kopf oder einem stark wachsenden Bruttoinlandsprodukt haben dagegen eher ein geringeres Konfliktrisiko. Auch die beiden Demokratisierungsvariablen erweisen sich als hochgradig signifikant und für den quadrierten Term ist das Vorzeichen wie erwartet negativ. Dies passt zu den bisherigen Befunden aus einem breiten Strang der empirischen Konfliktforschung, der eine kurvilineare Beziehung zwischen dem Demokratisierungsniveau eines Landes und dem Risiko gewalttätiger innerstaatlicher Konflikte untersucht.

Eine Ausnahme unter den Kontrollvariablen stellt der Export von Gütern und Dienstleistungen dar, der in keiner der drei Gleichungen ein konventionelles Signifikanzniveau erreicht. Die in der Literatur berichtete negative Beziehung zwischen Handelsverflechtung und Konfliktrisiko (Barbieri/Reuveny 2005; Martin et al. 2008; Goldstone et al. 2000) kann mit den Modellen nicht bestätigt werden.

\subsection{Kultur als Thema innerstaatlicher Konflikte}

Im zweiten Teil der Analyse gehen wir der Frage nach, wann Kultur zum Thema innerstaatlicher Konflikte wird. Wir verengen dazu den Fokus auf ausschließlich innerstaatliche Kulturkonflikte und lassen alle Auseinandersetzungen außen vor, in denen Sprache, Religion und Historizität kein prominentes Thema darstellen. Dabei ergibt sich ein zu den bisherigen Befunden ähnliches Muster, das jedoch auch eine Reihe von Abweichungen aufweist (Tabelle 5). 
Tabelle 5: Bestimmungsfaktoren innerstaatlicher Konfliktwahrscheinlichkeit 1950 bis 2005 (kulturelle Konflikte; binäre logistische Regression)

\begin{tabular}{|c|c|c|c|}
\hline & $\begin{array}{c}(1) \\
\text { CONIS }(3,4,5)\end{array}$ & $\begin{array}{c}(2) \\
\text { CONIS }(3,4,5)\end{array}$ & $\begin{array}{c}(3) \\
\text { CONIS }(3,4,5)\end{array}$ \\
\hline Konstante & $\begin{array}{c}-9,533 \\
(0,589)^{* * *}\end{array}$ & $\begin{array}{c}-10,128 \\
(0,586)^{* * *}\end{array}$ & $\begin{array}{c}-10,243 \\
(0,593)^{* * *}\end{array}$ \\
\hline Bevölkerung (logarithmiert) & $\begin{array}{c}0,733 \\
(0,038)^{* * *}\end{array}$ & $\begin{array}{c}0,812 \\
(0,038)^{* * *}\end{array}$ & $\begin{array}{c}0,767 \\
(0,038)^{* * *}\end{array}$ \\
\hline $\begin{array}{l}\text { Säuglingssterblichkeit } \\
\text { (pro } 1000 \text { Geburten, UNWPP) }\end{array}$ & $\begin{array}{c}0,002 \\
(0,001)\end{array}$ & $\begin{array}{c}0,009 \\
(0,001)^{* * *}\end{array}$ & $\begin{array}{c}0,005 \\
(0,001)^{* * *}\end{array}$ \\
\hline $\begin{array}{l}\text { Kultivierbare Landfläche } \\
\text { (Hektar pro Person, WDI) }\end{array}$ & $\begin{array}{c}-0,289 \\
(0,138)^{* *}\end{array}$ & $\begin{array}{c}-0,182 \\
(0,140)\end{array}$ & $\begin{array}{c}-0,246 \\
(0,133)^{*}\end{array}$ \\
\hline $\begin{array}{l}\text { Wachst. BIP/Kopf, int. US\$, PPP, konst. Prei- } \\
\text { se (Penn World Tables) }\end{array}$ & $\begin{array}{l}-0,005 \\
(0,007)\end{array}$ & $\begin{array}{l}-0,008 \\
(0,007)\end{array}$ & $\begin{array}{l}-0,007 \\
(0,007)\end{array}$ \\
\hline $\begin{array}{l}\text { Export von Gütern und Dienstleistungen (in } \\
\text { Prozent des BIP, WDI) }\end{array}$ & $\begin{array}{c}0,008 \\
(0,003)^{* *}\end{array}$ & $\begin{array}{c}0,021 \\
(0,003)^{* * *}\end{array}$ & $\begin{array}{c}0,014 \\
(0,003)^{* * *}\end{array}$ \\
\hline $\begin{array}{l}\text { Demokratisierungsgrad } \\
\text { (Polity IV: }-10 \text { bis }+10 \text { ) }\end{array}$ & $\begin{array}{c}0,038 \\
(0,008)^{* * *}\end{array}$ & $\begin{array}{c}0,042 \\
(0,008)^{* * *}\end{array}$ & $\begin{array}{c}0,042 \\
(0,008)^{* * *}\end{array}$ \\
\hline $\begin{array}{l}\text { Demokratisierungsgrad } \\
\text { (Polity IV, quadriert) }\end{array}$ & $\begin{array}{c}-0,004 \\
(0,002)^{* *}\end{array}$ & $\begin{array}{c}-0,004 \\
(0,002)^{* * *}\end{array}$ & $\begin{array}{c}-0,004 \\
(0,002)^{* *}\end{array}$ \\
\hline $\begin{array}{l}\text { Nettomigration pro } 1000 \text { Einw. } \\
\text { (positive Werte = Immigration) }\end{array}$ & $\begin{array}{l}-0,010 \\
(0,008)\end{array}$ & $\begin{array}{c}0,001 \\
(0,007)\end{array}$ & $\begin{array}{l}-0,002 \\
(0,008)\end{array}$ \\
\hline $\begin{array}{l}\text { Youth Bulge: Anteil der 15-24-jährigen Män- } \\
\text { ner an Männern über } 14 \text { Jahre }\end{array}$ & $\begin{array}{c}0,006 \\
(0,010)\end{array}$ & $\begin{array}{c}0,012 \\
(0,010)\end{array}$ & $\begin{array}{c}0,019 \\
(0,010)^{*}\end{array}$ \\
\hline Index der sprachlichen Fraktionalisierung & $\begin{array}{c}1,645 \\
(0,171)^{* * *}\end{array}$ & & \\
\hline Index der religiösen Fraktionalisierung & & $\begin{array}{c}-1,118 \\
(0,225)^{* * *}\end{array}$ & \\
\hline Index der kulturellen Fraktionalisierung & & & $\begin{array}{c}0,979 \\
(0,229)^{* * *} \\
\end{array}$ \\
\hline Pseudo- $R^{2}$ nach Nagelkerke & 0,265 & 0,245 & 0,243 \\
\hline Einbezogene Fälle $(\mathrm{N})$ & 4427 & 4421 & 4421 \\
\hline
\end{tabular}

Anmerkungen: siehe Tabelle 4.

Erneut ist die Konfliktwahrscheinlichkeit höher, wenn eine starke sprachliche Fraktionalisierung vorliegt. Im Gegensatz zur aufgeführten Betrachtung aller Konflikte erweist sich der kombinierte Index kultureller Fraktionalisierung im Hinblick auf kulturelle Konflikte jetzt allerdings als klar signifikant und er hat auch das theoretisch erwartete positive Vorzeichen: Kulturkonflikte sind demnach in Ländern mit einer hohen kulturellen Fraktionalisierung wahrscheinlicher als in homogenen Gesell- 
schaften. Das Vorzeichen für den Index der religiösen Fraktionalisierung weicht hingegen erneut von den oben formulierten theoretischen Erwartungen ab. Dieser erklärungsbedürftige Befund wird im Abschnitt 4.5 näher thematisiert. Dass der Koeffizient zwischen den Modellen auch in seiner Größe stark abnimmt, könnte analog zur sprachlichen Fraktionalisierung darauf zurückzuführen sein, dass sich die Effekte religiöser Fraktionalisierung vor allem in Form eines erhöhten Risikos für mittlere Konfliktniveaus äußern.

Bei den Kontrollgrößen werden auch hier verschiedene Indikatoren insignifikant. Insbesondere das Wirtschaftswachstum, aber auch die Migrationsrate und die kultivierbare Landfläche verlieren an Bedeutung. Sie sind schwächer und weniger signifikant mit dem Risiko eines kulturellen Konflikts assoziiert. Aber auch der Anteil junger Männer ist in zwei Gleichungen nicht mehr statistisch signifikant. Weiter unten soll deshalb gefragt werden, ob hier vielleicht Interaktionseffekte zwischen dem Anteil junger Männer und der kulturellen Fraktionalisierung eines Landes (in seinen verschiedenen Ausprägungen) bestehen. Auffällig ist zudem, dass der Koeffizient für den Export von Gütern und Dienstleistungen signifikant ist, wenn man ausschließlich kulturelle Konflikte betrachtet - allerdings widerspricht das Vorzeichen den theoretischen Erwartungen.

Einen möglichen Ansatzpunkt für Kritik stellt die potenzielle Nähe zwischen abhängiger Variable und den drei unabhängigen kulturellen Einflussgrößen dar. Dem möglichen Einwand einer Tautologie sei jedoch entgegnet, dass, wie bereits ausgeführt wurde, Ursache und Thema von Konflikten nicht identisch sein müssen, mit anderen Worten: Kulturkonflikte können nicht-kulturelle Auslöser haben und kulturelle Auslöser können zu nicht-kulturell konnotierten Konflikten führen. Damit besteht von theoretischer Seite her keine zwingende Verbindung zwischen abhängiger und unabhängiger Größe. Darüber hinaus liegt auch in den Erhebungskriterien (Bevölkerungsanteile zur Indexerhebung gegenüber Thematisierungen zur Konflikteinstufung) keine konzeptionelle Beziehung vor - wie groß eine Bevölkerungsgruppe tatsächlich ist, hat kaum Einfluss darauf, wie sehr sie als »anders « und die eigene Identität berührend dargestellt wird.

\subsection{Kultur als Ursache zwischenstaatlicher Konflikte}

Nachdem wir die Bedeutung des kulturellen setup eines Landes für dessen innerstaatliche Konfliktbelastung untersucht haben, gehen wir nun der Frage nach, inwieweit kulturelle Indikatoren ein signifikanter Prädiktor für die zwischenstaatliche Konfliktbelastung eines Landes im Allgemeinen sind (Kultur als Ursache). Die Ergebnisse der logistischen Regression zur Wahrscheinlichkeit zwischenstaatlicher Konflikte sind in Tabelle 6 dargestellt. 
Tabelle 6: Bestimmungsfaktoren zwischenstaatlicher Konfliktwahrscheinlichkeit 1950 bis 2005 (alle Konflikte; binäre logistische Regression)

\begin{tabular}{|c|c|c|c|}
\hline & $\begin{array}{c}(1) \\
\text { CONIS }(3,4,5)\end{array}$ & $\begin{array}{c}(2) \\
\text { CONIS }(3,4,5)\end{array}$ & $\begin{array}{c}(3) \\
\text { CONIS }(3,4,5)\end{array}$ \\
\hline Konstante & $\begin{array}{c}-8,338 \\
(0,691)^{* * *}\end{array}$ & $\begin{array}{c}-9,176 \\
(0,702)^{* * *}\end{array}$ & $\begin{array}{c}-8,767 \\
(0,696)^{* * *}\end{array}$ \\
\hline Bevölkerung (logarithmiert) & $\begin{array}{c}0,531 \\
(0,041)^{* * *}\end{array}$ & $\begin{array}{c}0,579 \\
(0,041)^{* * *}\end{array}$ & $\begin{array}{c}0,533 \\
(0,041)^{* * *}\end{array}$ \\
\hline $\begin{array}{l}\text { Säuglingssterblichkeit } \\
\text { (pro } 1000 \text { Geburten, UNWPP) }\end{array}$ & $\begin{array}{c}-0,006 \\
(0,002)^{* * *}\end{array}$ & $\begin{array}{l}-0,002 \\
(0,002)\end{array}$ & $\begin{array}{c}-0,006 \\
(0,002)^{* * *}\end{array}$ \\
\hline $\begin{array}{l}\text { Kultivierbare Landfläche } \\
\text { (Hektar pro Person, WDI) }\end{array}$ & $\begin{array}{c}-1,341 \\
(0,273)^{* * *}\end{array}$ & $\begin{array}{c}-1,208 \\
(0,263)^{* * *}\end{array}$ & $\begin{array}{c}-1,275 \\
(0,265)^{* * *}\end{array}$ \\
\hline $\begin{array}{l}\text { Wachst. BIP/Kopf, int. US\$, PPP, konst. Prei- } \\
\text { se (Penn World Tables) }\end{array}$ & $\begin{array}{c}0,000 \\
(0,007)\end{array}$ & $\begin{array}{l}-0,005 \\
(0,008)\end{array}$ & $\begin{array}{l}-0,003 \\
(0,008)\end{array}$ \\
\hline $\begin{array}{l}\text { Export von Gütern und Dienstleistungen (in } \\
\text { Prozent des BIP, WDI) }\end{array}$ & $\begin{array}{c}0,001 \\
(0,004)\end{array}$ & $\begin{array}{c}0,007 \\
(0,004)^{* *}\end{array}$ & $\begin{array}{c}0,001 \\
(0,004)\end{array}$ \\
\hline $\begin{array}{l}\text { Demokratisierungsgrad } \\
\text { (Polity IV: }-10 \text { bis }+10)\end{array}$ & $\begin{array}{c}-0,033 \\
(0,009)^{* * *}\end{array}$ & $\begin{array}{c}-0,029 \\
(0,009)^{* * *}\end{array}$ & $\begin{array}{c}-0,031 \\
(0,009)^{* * *}\end{array}$ \\
\hline $\begin{array}{l}\text { Demokratisierungsgrad } \\
\text { (Polity IV, quadriert) }\end{array}$ & $\begin{array}{c}-0,004 \\
(0,002)^{*}\end{array}$ & $\begin{array}{c}-0,004 \\
(0,002)^{*}\end{array}$ & $\begin{array}{c}-0,003 \\
(0,002)^{*}\end{array}$ \\
\hline $\begin{array}{l}\text { Nettomigration pro } 1000 \text { Einw. } \\
\text { (positive Werte = Immigration) }\end{array}$ & $\begin{array}{c}0,004 \\
(0,007)\end{array}$ & $\begin{array}{c}0,011 \\
(0,007)^{*}\end{array}$ & $\begin{array}{c}0,007 \\
(0,007)\end{array}$ \\
\hline $\begin{array}{l}\text { Youth Bulge: Anteil der 15-24-jährigen Män- } \\
\text { ner an Männern über } 14 \text { Jahre }\end{array}$ & $\begin{array}{c}0,044 \\
(0,013)^{* * *}\end{array}$ & $\begin{array}{c}0,056 \\
(0,013)^{* * *}\end{array}$ & $\begin{array}{c}0,053 \\
(0,013)^{* * *}\end{array}$ \\
\hline Index der sprachlichen Fraktionalisierung & $\begin{array}{c}1,470 \\
(0,203)^{* * *}\end{array}$ & & \\
\hline Index der religiösen Fraktionalisierung & & $\begin{array}{c}0,816 \\
(0,256)^{* * *}\end{array}$ & \\
\hline Index der kulturellen Fraktionalisierung & & & $\begin{array}{c}1,971 \\
(0,286)^{* * *} \\
\end{array}$ \\
\hline Pseudo- $\mathrm{R}^{2}$ nach Nagelkerke & 0,191 & 0,171 & 0,188 \\
\hline Einbezogene Fälle (N) & 4427 & 4421 & 4421 \\
\hline
\end{tabular}

Anmerkungen: siehe Tabelle 4.

Wie in Abschnitt 4.1 ist die abhängige Variable erneut die allgemeine Belastung eines Landes durch Konflikte, ganz gleich ob mit oder ohne kulturelle Thematisierung. In den entsprechenden Hypothesen war im theoretischen Teil die Erwartung formuliert worden, dass sprachliche, religiöse und kulturelle Fraktionalisierung zwischen Staaten keine Rolle spielt, da sich die Konfliktgegenstände auf dieser Ebene von denen innerstaatlicher Auseinandersetzungen unterscheiden. Akzeptiert man das Pseudo- ${ }^{2}$ 
nach Nagelkerke als Näherungsgröße für die Erklärungskraft der Modelle, fällt zuerst auf, dass die Gleichungen offenbar weniger imstande sind, die Konfliktbelastung auf zwischenstaatlicher als auf innerstaatlicher Ebene zu erklären. Dennoch sind alle drei kulturellen Faktoren hochsignifikant - und anders als bei den obenstehenden Modellen besitzen die Koeffizienten diesmal alle das positive Vorzeichen, das eigentlich für innerstaatliche Konflikte erwartet worden war. Auffällig ist zudem, dass der Grad der sprachlichen Fraktionalisierung einen deutlich größeren Regressionsparameter aufweist als im Modell für das innerstaatliche Konfliktpanorama in Tabelle 4. Dies deutet darauf hin, dass sprachliche Fraktionalisierung auf zwischenstaatlicher Ebene eine größere Rolle für die Konfliktwahrscheinlichkeit spielt als auf der innerstaatlichen.

Der Blick auf die Kontrollvariablen zeigt, dass verschiedene Erklärungsfaktoren, die für die innerstaatliche Konfliktwahrscheinlichkeit relevant waren, bei der Analyse zwischenstaatlicher Auseinandersetzungen an Signifikanz verlieren. Hierzu zählt das Wirtschaftswachstum eines Landes, aber auch der Grad der Demokratisierung. Migration und Export sind ebenfalls von geringerer Bedeutung. Die Bevölkerungsgröße, youth bulges und die Menge kultivierbaren Landes pro Kopf haben hingegen weiterhin signifikante Parameter, die in die theoretisch erwartete Richtung weisen. Das Vorzeichen für das Entwicklungsniveau eines Landes - gemessen als Säuglingssterblichkeitsrate - hingegen ist negativ und zeigt damit in eine unerwartete Richtung. Dem in zwei Modellen signifikanten Koeffizienten zufolge sind Länder mit niedriger Säuglingssterblichkeit eher in zwischenstaatliche Konflikte verwickelt als andere Staaten. Die Befunde in Tabelle 6 führen damit zum Ergebnis, dass auch auf der zwischenstaatlichen Ebene die kulturelle Fraktionalisierung eine Rolle zu spielen scheint.

\subsection{Kultur als Thema zwischenstaatlicher Konflikte}

Im vierten Schritt der Analyse greifen wir jene zwischenstaatlichen Konflikte heraus, in denen Kultur ein hervorgehobenes Thema der Auseinandersetzung darstellte und prüfen, ob sich signifikante Erklärungsgrößen für kulturelle aufgeladene zwischenstaatliche Konflikte identifizieren lassen. Die Ergebnisse der statistischen Analyse sind in Tabelle 7 dargestellt. 
Tabelle 7: Bestimmungsfaktoren zwischenstaatlicher Konfliktwahrscheinlichkeit 1950 bis 2005 (kulturelle Konflikte; binäre logistische Regression)

\begin{tabular}{|c|c|c|c|}
\hline & $\begin{array}{c}(1) \\
\text { CONIS }(3,4,5)\end{array}$ & $\begin{array}{c}(2) \\
\text { CONIS }(3,4,5)\end{array}$ & $\begin{array}{c}(3) \\
\text { CONIS }(3,4,5)\end{array}$ \\
\hline Konstante & $\begin{array}{c}-8,468 \\
(0,970)^{* * *}\end{array}$ & $\begin{array}{c}-9,275 \\
(0,972)^{* * *}\end{array}$ & $\begin{array}{c}-9,284 \\
(0,974)^{* * *}\end{array}$ \\
\hline Bevölkerung (logarithmiert) & $\begin{array}{c}0,351 \\
(0,054)^{* * *}\end{array}$ & $\begin{array}{c}0,510 \\
(0,056)^{* * *}\end{array}$ & $\begin{array}{c}0,407 \\
(0,055)^{* * *}\end{array}$ \\
\hline $\begin{array}{l}\text { Säuglingssterblichkeit } \\
\text { (pro } 1000 \text { Geburten, UNWPP) }\end{array}$ & $\begin{array}{l}-0,004 \\
(0,002)^{*}\end{array}$ & $\begin{array}{c}0,006 \\
(0,002)^{* * *}\end{array}$ & $\begin{array}{c}0,000 \\
(0,002)\end{array}$ \\
\hline $\begin{array}{l}\text { Kultivierbare Landfläche } \\
\text { (Hektar pro Person, WDI) }\end{array}$ & $\begin{array}{c}-1,374 \\
(0,384)^{* * *}\end{array}$ & $\begin{array}{c}-1,439 \\
(0,405)^{* * *}\end{array}$ & $\begin{array}{c}-1,287 \\
(0,370)^{* * *}\end{array}$ \\
\hline $\begin{array}{l}\text { Wachst. BIP/Kopf, int. US\$, PPP, konst. Preise } \\
\text { (Penn World Tables) }\end{array}$ & $\begin{array}{l}-0,004 \\
(0,010)\end{array}$ & $\begin{array}{l}-0,009 \\
(0,011)\end{array}$ & $\begin{array}{l}-0,009 \\
(0,010)\end{array}$ \\
\hline $\begin{array}{l}\text { Export von Gütern und Dienstleistungen (in } \\
\text { Prozent des BIP, WDI) }\end{array}$ & $\begin{array}{c}-0,016 \\
(0,007)^{* *}\end{array}$ & $\begin{array}{c}0,004 \\
(0,006)\end{array}$ & $\begin{array}{l}-0,007 \\
(0,006)\end{array}$ \\
\hline $\begin{array}{l}\text { Demokratisierungsgrad } \\
\text { (Polity IV: }-10 \text { bis }+10 \text { ) }\end{array}$ & $\begin{array}{c}0,001 \\
(0,012)\end{array}$ & $\begin{array}{c}0,004 \\
(0,012)\end{array}$ & $\begin{array}{c}0,007 \\
(0,012)\end{array}$ \\
\hline $\begin{array}{l}\text { Demokratisierungsgrad } \\
\text { (Polity IV, quadriert) }\end{array}$ & $\begin{array}{c}0,010 \\
(0,003)^{* * *}\end{array}$ & $\begin{array}{c}0,009 \\
(0,003)^{* * *}\end{array}$ & $\begin{array}{c}0,010 \\
(0,003)^{* * *}\end{array}$ \\
\hline $\begin{array}{l}\text { Nettomigration pro } 1000 \text { Einw. } \\
\text { (positive Werte = Immigration) }\end{array}$ & $\begin{array}{c}0,022 \\
(0,008)^{* * *}\end{array}$ & $\begin{array}{c}0,024 \\
(0,007)^{* * *}\end{array}$ & $\begin{array}{c}0,025 \\
(0,008)^{* * *}\end{array}$ \\
\hline $\begin{array}{l}\text { Youth Bulge: Anteil der 15-24-jährigen Män- } \\
\text { ner an Männern über } 14 \text { Jahre }\end{array}$ & $\begin{array}{c}0,044 \\
(0,018)^{* *}\end{array}$ & $\begin{array}{c}0,040 \\
(0,018)^{* *}\end{array}$ & $\begin{array}{c}0,057 \\
(0,017)^{* * *}\end{array}$ \\
\hline Index der sprachlichen Fraktionalisierung & $\begin{array}{c}2,327 \\
(0,314)^{* * *}\end{array}$ & & \\
\hline Index der religiösen Fraktionalisierung & & $\begin{array}{c}-1,742 \\
(0,408)^{* * *}\end{array}$ & \\
\hline Index der kulturellen Fraktionalisierung & & & $\begin{array}{c}1,331 \\
(0,396)^{* * *}\end{array}$ \\
\hline Pseudo- $R^{2}$ nach Nagelkerke & 0,143 & 0,112 & 0,107 \\
\hline Einbezogene Fälle $(\mathrm{N})$ & 4427 & 4421 & 4421 \\
\hline
\end{tabular}

Anmerkungen: siehe Tabelle 4.

Erneut zeigt sich für den Index der sprachlichen Fraktionalisierung ein hochsignifikantes Ergebnis. Gegenüber den Modellen für die innerstaatlichen Kulturkonflikte ist der Koeffizient deutlich größer und er ist auch dann noch hochsignifikant, wenn man (hier nicht aufgeführt) nur Konflikte der Stufe 4 und 5 zusammenfasst. Die religiöse Fraktionalisierung ist dagegen erneut negativ mit der Konfliktwahrscheinlichkeit assoziiert, sie folgt somit dem bereits auf innerstaatlicher Ebene beobachteten Muster. 
Im Vergleich zu den Modellen für das zwischenstaatliche Konfliktpanorama ist dieser Vorzeichenwechsel der wohl auffallendste Unterschied, der mit der Veränderung der abhängigen Variable einhergeht. Anders als bei den innerstaatlichen Konflikten wiegt die starke Assoziation der sprachlichen Fraktionalisierung die umgekehrte Ausrichtung der religiösen Fraktionalisierung im zusammengefassten Index der kulturellen Fraktionalisierung mehr als auf - der Koeffizient in Modell 3 ist positiv und signifikant. Es fällt zudem auf, dass die Nettomigrationsrate für kulturelle Konflikte einen signifikanten Prädiktor darstellt - eine Beziehung, die für das allgemeine Konfliktrisiko nicht besteht.

\subsection{Erweiterung der Erklärungsmodelle: Nichtlinearität und Interaktionen bei innerstaatlichen Konflikten}

Die bisherige Untersuchung hat für die religiöse Fraktionalisierung teilweise ein unerwartetes Ergebnis geliefert. Auf innerstaatlicher Ebene ist der entsprechende Index signifikant mit einer Senkung des Konfliktrisikos verbunden, auf zwischenstaatlicher Ebene zeigt sich dieselbe Assoziation für die Kulturkonflikte, nicht aber für das gesamte Konfliktpanorama. Warum könnte die religiöse Fraktionalisierung diesen Effekt aufweisen? Eine mögliche Erklärung hierfür könnten systematische Verzerrungen des Datenbestandes in Verbindung mit einem Endogenitätsproblem sein. Die Qualität und Verlässlichkeit statistischer Erhebungen ist in den hier untersuchten Ländern sehr unterschiedlich. Während die meisten Industrieländer über ausgefeilte Methoden der Datensammlung verfügen, stehen für Entwicklungsländer - noch dazu wenn diese von Konflikten betroffen sind - oft nur rudimentäre Schätzungen zur Verfügung. Wegen der damit verbundenen Ungenauigkeit ist es sehr wahrscheinlich, dass in den wohlhabenderen (und tendenziell weniger von Konflikten betroffenen) Industrieländern eine höhere Anzahl von religiösen Gruppen gezählt wird als in Entwicklungsländern. Daher dürfte der Index in letzteren Staaten tendenziell zu niedrig ausfallen, also eine geringere Fraktionalisierung ausweisen als tatsächlich besteht.

Neben Bedenken im Hinblick auf die Qualität der Datengrundlage könnte aber auch ein Endogenitätsrisiko zum unerwarteten Verhalten des Indikators beitragen. Alberto Alesina, Arnaud Devleeschauwer, William Easterly, Sergio Kurlat und Romain Wacziarg $(2003$ : 165, 167) beispielsweise finden in ihrer Untersuchung der Beziehung religiöser, ethnischer und sprachlicher Fraktionalisierung zum langfristigen Wirtschaftswachstum keine konsistenten Effekte religiöser Fraktionalisierung. Sie interpretieren diesen Befund dahingehend, dass Religion im Gegensatz zu den anderen erhobenen Merkmalen Ethnie (die oft an die Hautfarbe einer Person anknüpft) und Sprache ein persönliches Merkmal ist, das bei sonst drohender Repression schlicht geleugnet werden kann. Da sich die eigene Religion zudem im Lauf des Lebens ändern kann, vermuten sie, dass entwickelte Länder einen höheren Grad re- 
ligiöser Fraktionalisierung aufweisen könnten, weil sie schlicht toleranter sind (Alesina et al. 2003: 167)..$^{10}$

Ein dritter Grund für das unerwartete Vorzeichen könnte aber auch eine nichtlineare Beziehung zwischen unabhängiger und abhängiger Größe sein. Wie könnte eine solche Verbindung aussehen? Kern der bisherigen Hypothesen war, dass sich mit der Fraktionalisierung einer Gesellschaft ihr kulturelles Strukturmuster verändert, da die Zahl der in ihr auftretenden Akteure zunimmt. Vor allem für Bereiche hoher Fraktionalisierung erscheint jedoch auch die Vermutung plausibel, dass zwar die Wahrscheinlichkeit für das Auftreten von Akteuren mit steigender Fraktionalisierung weiter zunimmt, dass sich aber ab einem gewissen Grad aufgrund der Struktur dieser Akteure das Konfliktrisiko nicht weiter erhöht bzw. dass es sogar sinkt. Mit anderen Worten: Zwar sind mit steigender Fraktionalisierung in jedem Fall auch mehr Akteure zu erwarten, es besteht jedoch die Möglichkeit, dass damit nicht mehr unbedingt auch ein Anstieg des Konfliktrisikos einhergeht, weshalb als Konkretisierung der bisherigen Hypothesen im Folgenden getestet wird, ob die Verbindung zwischen religiöser und sprachlicher Fraktionalisierung als nichtlinear weiter qualifizierbar ist.

Annahmen zu einer nichtlinearen Wirkung kultureller (meist ethnischer) Fraktionalisierung auf das Konfliktniveau eines Landes finden sich in der Literatur an mehreren Stellen. So argumentiert beispielsweise Tanja Ellingsen (2000: 232), dass ethnisch inhomogene Länder angesichts nicht deckungsgleicher Grenzen von Staat und Ethnie Probleme haben dürften, einen Demos zu konstruieren, welcher der politischen Gemeinschaft Legitimität verleihen könnte. Folglich sollte das entsprechende Land einem erhöhten Konfliktrisiko ausgesetzt sein: »If fragmented societies find it difficult to define >the people<, one would expect the likelihood of domestic conflict to increase the more fragmented a society«. Umgekehrt kann aber auch das Risiko gewaltsamer Aufstände zunehmen, wenn eine Minderheit von adäquaten Zugängen zu Schlüsselpositionen innerhalb einer Gesellschaft ferngehalten wird - eine Situation, die vor allem dann zu Konflikten führen dürfte, wenn die sich gegenüberstehenden Gruppen fast gleich groß sind, da hier die Chancen auf eine erfolgreiche Rebellion am besten sind. Da sich beide Überlegungen in ihrem Extrem widersprechen, fasst Ellingsen diese als komplementäre Erklärungsansätze auf und argumentiert, dass vor allem in Gesellschaften mittlerer Fraktionalisierung ein erhöhtes Konfliktrisiko besteht, weil hier die dominante Gruppe »large but not too large« (Ellingsen 2000: 233) ist und bei steigender Fraktionalisierung entweder die Wahrscheinlichkeit einer Diskriminierung anderer Gruppen nachlässt bzw. bei sinkender Fraktionalisierung die Möglichkeit einer Minderheit zur Rebellion zurückgeht.

Ebenfalls mit Blick auf die ethnische Fraktionalisierung vermuten James Fearon und David Laitin (2003: 78) im Anschluss an Donald Horowitz (1985), dass die politischen Prozesse sehr homogener oder sehr heterogener Länder einander insofern

10 Ein der Erhebung der religiösen Bevölkerungsdaten vergleichbares Problem besteht bei den sprachbezogenen Daten nicht. Die verfügbaren Informationen sind hier detaillierter und zuverlässiger. Dieser Umstand ist quellenbedingt, liegt aber auch im kommunikativen »Wesen« des Sprachlichen, das deutlich weniger »personalisierbar« ist als religiöse Anschauungen. 
strategisch ähneln könnten, als dass in beiden Fällen Koalitionen kleiner Gruppen notwendig sind, um Einfluss zu gewinnen. Wegen der großen Zahl an potentiellen Variationen sei daher in heterogenen Ländern eher dann mit einem erhöhten Konfliktrisiko zu rechnen, wenn eine relativ große Minderheit einer dominanten Mehrheit gegenüberstehe, aber keine Aussicht auf eine Machtübernahme besitze. Collier und Hoeffler gehen davon aus, dass eine hohe soziale Fraktionalisierung das Risiko gewaltsamer innerstaatlicher Konfrontationen senkt, da auf der einen Seite die Koordinationskosten für Rebellen zunehmen, gleichzeitig aber die Größe ihres Rekrutierungspotentials (die jeweilige Ethnie) zurückgeht (Collier/Hoeffler 1998: 566-567).

Im Hinblick auf den Grad religiöser Fraktionalisierung stellen Fearon und Laitin (2003), Collier und Hoeffler (2004) sowie José Montalvo und Marta Reynal-Querol (2005) fest, dass dieser auf das Bürgerkriegsrisiko einer Gesellschaft keinen Einfluss ausübt (vgl. die Übersicht bei de Juan/Hasenclever 2008). Wir gehen davon aus, dass sich dieser Befund im Rahmen des vorliegenden Untersuchungsansatzes aus den genannten Gründen nicht bestätigen wird. Nicht nur bei einer mittleren sprachlichen, sondern auch bei einer mittleren religiösen Fraktionalisierung sollte eine erhöhte Wahrscheinlichkeit innerstaatlicher Konflikte zu erwarten sein. Im Folgenden sind daher zwei Hypothesen zur Nichtlinearität (nl) zu testen, die jedoch in Form von Interaktionstermen mit dem Faktor youth bulge überprüft werden (vgl. Tabelle 8):

- Hypothese 1(nl): Länder mit einem mittleren Grad religiöser Fraktionalisierung haben ein gegenüber anderen Ländern erhöhtes Risiko innerstaatlicher Konflikte.

- Hypothese 2(nl): Länder mit einem mittleren Grad sprachlicher Fraktionalisierung haben ein gegenüber anderen Ländern erhöhtes Risiko innerstaatlicher Konflikte.

Interaktionseffekte ergeben dann Sinn, wenn die Ausprägung einer Variable darüber entscheidet, ob die Wirkung einer anderen Variable verstärkt oder abgeschwächt wird. Im Hinblick auf kulturelle Einflussfaktoren erscheinen youth bulges insofern als ein naheliegender Kandidat, als dass Huntington (1997) ihnen eine zentrale Rolle als Motor seiner »Kulturkampfthese« zukommen lässt: Das Wiederaufkommen islamischer Werte und in dessen Gefolge auch islamistischer Gewalt ist demnach neben traditionellen Mittelschichten und Migranten vor allem durch junge, oftmals gebildete Menschen getragen, die den Kern der militanten Protagonisten stellen (Huntington 1997: 156, 174). Die hohe Beteiligung muslimischer Länder an interkulturellen Bruchlinienkriegen erklärt er damit, dass hier eine starke Bevölkerungsexpansion einen kritischen Populationsdruck hervorgerufen hat:

»Die Bevölkerungsexplosion in muslimischen Gesellschaften und das riesige Reservoir an oft beschäftigungslosen Männern zwischen 15 und 30 sind eine natürliche Quelle der Instabilität und der Gewalt innerhalb des Islam wie gegen Nichtmuslime. Welche anderen Gründe auch sonst noch mitspielen mögen, dieser Faktor allein erklärt zu einem großen Teil die muslimische Gewalt der achtziger und neunziger Jahre« (Huntington 1997: 433).

Konflikte (die teilweise auch als Kulturkonflikte gedeutet und konstruiert werden können) könnten im Licht dieser Überlegungen möglicherweise deutlich durch demographische Ursachen mit befördert werden, während Sprache, Religion, Ethnie oder Ideologie vielleicht nur einen vordergründigen Auslöser oder zumindest einen 
vor allem im Verbund mit demographischen Verwerfungen entscheidenden Faktor darstellen.

Tabelle 8 fasst die Ergebnisse für innerstaatliche Konflikte (sowohl für das gesamte Konfliktpanorama als auch für Kulturkonflikte allein) zusammen, wobei religiöse und sprachliche Fraktionalisierung jeweils als nichtlineare Einflussgrößen sowie zusätzlich deren Interaktion mit dem Faktor youth bulge berücksichtigt wurden. Für die Modelle wurden die einzelnen Länder dann mit einer Dummy-Variable markiert, wenn der jeweilige Fraktionalisierungsindex im Intervall von 0,3 bis 0,7 lag (die Gesamtspannweite reicht von 0 bis 1) und so einen mittleren Fraktionalisierungsgrad anzeigte. Der Interaktionsterm wird berechnet, indem diese Dummy-Variable anschließend mit der youth bulge-Variable multipliziert wurde.

Tabelle 8: Nichtlinearität und Interaktionseffekte der kulturellen Fraktionalisierung zur Erklärung innerstaatlicher Konfliktwahrscheinlichkeit 1950 bis 2005

\begin{tabular}{|c|c|c|c|c|}
\hline & $\begin{array}{c}\text { Alle } \\
\text { Konflikte } \\
(1) \\
\text { CONIS } \\
(3,4,5)\end{array}$ & $\begin{array}{c}\text { Kulturkon- } \\
\text { flikte } \\
(2) \\
\text { CONIS } \\
(3,4,5)\end{array}$ & $\begin{array}{c}\text { Alle } \\
\text { Konflikte } \\
(3) \\
\text { CONIS } \\
(3,4,5)\end{array}$ & $\begin{array}{c}\text { Kulturkonflik- } \\
\text { te } \\
(4) \\
\text { CONIS }(3,4,5)\end{array}$ \\
\hline Konstante & $\begin{array}{c}-8,703 \\
(0,534)^{* * *}\end{array}$ & $\begin{array}{c}-10,358 \\
(0,606)^{* * *}\end{array}$ & $\begin{array}{c}-8,492 \\
(0,532)^{* * *}\end{array}$ & $\begin{array}{c}-10,323 \\
(0,602)^{* * *}\end{array}$ \\
\hline Bevölkerung (logarithmiert) & $\begin{array}{c}0,597 \\
(0,032)^{* * *}\end{array}$ & $\begin{array}{c}0,778 \\
(0,037)^{* * *}\end{array}$ & $\begin{array}{c}0,594 \\
(0,032)^{* * *}\end{array}$ & $\begin{array}{c}0,781 \\
(0,037)^{* * * *}\end{array}$ \\
\hline $\begin{array}{l}\text { Säuglingssterblichkeit } \\
\text { (pro } 1000 \text { Geburten, UNWPP) }\end{array}$ & $\begin{array}{c}0,004 \\
(0,001)^{* * *}\end{array}$ & $\begin{array}{c}0,008 \\
(0,001)^{* * *}\end{array}$ & $\begin{array}{c}0,002 \\
(0,001)^{* *}\end{array}$ & $\begin{array}{c}0,007 \\
(0,001)^{* * *}\end{array}$ \\
\hline $\begin{array}{l}\text { Kultivierbare Landfläche } \\
\text { (Hektar pro Person, WDI) }\end{array}$ & $\begin{array}{c}-0,541 \\
(0,137)^{* * *}\end{array}$ & $\begin{array}{l}-0,212 \\
(0,132)\end{array}$ & $\begin{array}{c}-0,597 \\
(0,140)^{* * *}\end{array}$ & $\begin{array}{l}-0,219 \\
(0,135)\end{array}$ \\
\hline $\begin{array}{l}\text { Wachst. BIP/Kopf, int. US\$, PPP, } \\
\text { konst. Preise (Penn World Tables) }\end{array}$ & $\begin{array}{c}-0,017 \\
(0,006)^{* * *}\end{array}$ & $\begin{array}{l}-0,009 \\
(0,007)\end{array}$ & $\begin{array}{c}-0,017 \\
(0,006)^{* * *}\end{array}$ & $\begin{array}{l}-0,009 \\
(0,007)\end{array}$ \\
\hline $\begin{array}{l}\text { Export von Gütern und Dienstleistun- } \\
\text { gen (in Prozent des BIP, WDI) }\end{array}$ & $\begin{array}{c}0,001 \\
(0,003)\end{array}$ & $\begin{array}{c}0,017 \\
(0,003)^{* * *}\end{array}$ & $\begin{array}{c}0,000 \\
(0,003)\end{array}$ & $\begin{array}{c}0,017 \\
(0,003)^{* * *}\end{array}$ \\
\hline $\begin{array}{l}\text { Demokratisierungsgrad } \\
\text { (Polity IV: }-10 \text { bis }+10)\end{array}$ & $\begin{array}{c}0,054 \\
(0,007)^{* * *}\end{array}$ & $\begin{array}{c}0,041 \\
(0,008)^{* * *}\end{array}$ & $\begin{array}{c}0,049 \\
(0,007)^{* * *}\end{array}$ & $\begin{array}{c}0,040 \\
(0,008)^{* * *}\end{array}$ \\
\hline $\begin{array}{l}\text { Demokratisierungsgrad } \\
\text { (Polity IV, quadriert) }\end{array}$ & $\begin{array}{c}-0,008 \\
(0,001)^{* * *}\end{array}$ & $\begin{array}{c}-0,004 \\
(0,002)^{* * *}\end{array}$ & $\begin{array}{c}-0,008 \\
(0,001)^{* * *}\end{array}$ & $\begin{array}{c}-0,005 \\
(0,002)^{* * *}\end{array}$ \\
\hline $\begin{array}{l}\text { Nettomigration pro } 1000 \text { Einw. } \\
\text { (positive Werte = Immigration) }\end{array}$ & $\begin{array}{c}-0,012 \\
(0,006)^{* *}\end{array}$ & $\begin{array}{c}0,000 \\
(0,007)\end{array}$ & $\begin{array}{c}-0,011 \\
(0,006)^{*}\end{array}$ & $\begin{array}{c}0,001 \\
(0,007)\end{array}$ \\
\hline $\begin{array}{l}\text { Youth Bulge: Anteil der 15-24-jährigen } \\
\text { Männer an Männern über 14 Jahre } \\
\text { (UNWPP) }\end{array}$ & $\begin{array}{c}0,081 \\
(0,010)^{* * *}\end{array}$ & $\begin{array}{c}0,027 \\
(0,011)^{* *}\end{array}$ & $\begin{array}{c}0,076 \\
(0,010)^{* * *}\end{array}$ & $\begin{array}{c}0,024 \\
(0,011)^{* *}\end{array}$ \\
\hline
\end{tabular}




\begin{tabular}{|c|c|c|c|c|}
\hline & $\begin{array}{c}\text { Alle } \\
\text { Konflikte } \\
(1) \\
\text { CONIS } \\
(3,4,5)\end{array}$ & $\begin{array}{c}\text { Kulturkon- } \\
\text { flikte } \\
(2) \\
\text { CONIS } \\
(3,4,5)\end{array}$ & $\begin{array}{c}\text { Alle } \\
\text { Konflikte } \\
(3) \\
\text { CONIS } \\
(3,4,5)\end{array}$ & $\begin{array}{c}\text { Kulturkonflik- } \\
\text { te } \\
(4) \\
\text { CONIS }(3,4,5)\end{array}$ \\
\hline $\begin{array}{l}\text { Dummy für mittlere religiöse Fraktio- } \\
\text { nalisierung }(0=\text { niedrige und hohe } \\
\text { Fraktionalisierung })\end{array}$ & $\begin{array}{c}1,209 \\
(0,387)^{* * *}\end{array}$ & $\begin{array}{c}0,841 \\
(0,411)^{* *}\end{array}$ & & \\
\hline $\begin{array}{l}\text { Interaktionsterm: Dummy mittlere reli- } \\
\text { giöse Fraktionalisierung und männl. } \\
\text { Youth Bulge }\end{array}$ & $\begin{array}{c}-0,047 \\
(0,012)^{* * *}\end{array}$ & $\begin{array}{c}-0,029 \\
(0,013)^{* *}\end{array}$ & & \\
\hline $\begin{array}{l}\text { Dummy für mittlere sprachliche Frak- } \\
\text { tionalisierung }(0=\text { niedrige und hohe } \\
\text { Fraktionalisierung })\end{array}$ & & & $\begin{array}{c}1,181 \\
(0,390)^{* * *}\end{array}$ & $\begin{array}{c}0,867 \\
(0,415)^{* *}\end{array}$ \\
\hline $\begin{array}{l}\text { Interaktionsterm: Dummy mittlere } \\
\text { sprachliche Fraktionalisierung und } \\
\text { männl. Youth Bulge }\end{array}$ & & & $\begin{array}{c}-0,041 \\
(0,012)^{* * *}\end{array}$ & $\begin{array}{c}-0,024 \\
(0,013)^{*}\end{array}$ \\
\hline Pseudo- $\mathrm{R}^{2}$ nach Nagelkerke & 0,246 & 0,236 & 0,241 & 0,236 \\
\hline Einbezogene Fälle (N) & 4427 & 4427 & 4427 & 4427 \\
\hline
\end{tabular}

Anmerkungen: siehe Tabelle 4.

Fokussiert man auf die Länder mit mittlerer sprachlicher und religiöser Fraktionalisierung, so zeigt sich eine deutliche Veränderung der Ergebnisse. Als erstes fällt auf, dass in allen vier Modellen die Dummy-Variable und damit der Konditionaleffekt für eine mittlere Fraktionalisierung positiv und signifikant ist - bei einem gegebenen durchschnittlichen Anteil junger Männer in der Bevölkerung haben Länder mit einem sprachlichen oder religiösen Fraktionalisierungsgrad zwischen 0,3 und 0,7 demnach ein höheres Risiko für innerstaatliche Konflikte, unabhängig davon, ob es sich dabei um politische Konflikte allgemein oder um kulturell eingefärbte Auseinandersetzungen im Besonderen handelt. Insofern erscheinen die ersten Befunde für den Faktor Religion in einem neuen Licht: Das Risiko eines Konfliktes (gleich ob kulturell konnotiert oder nicht) in fraktionalisierten Gesellschaften geht also weniger von religiöser oder sprachlicher Zersplitterung allgemein aus, als vielmehr von einer Situation, in der sich wenige und ungefähr gleich große Gruppen gegenüberstehen. Deutlich überraschender mag hingegen das Vorzeichen für die vier Interaktionseffekte mit der youth bulge-Variable sein: Zwar erhöht der Anteil junger Männer in allen untersuchten Ländern das Konfliktrisiko, der Effekt ist jedoch in Ländern mit einem mittleren 
Fraktionalisierungsniveau spürbar geringer als in Ländern mit einer niedrigen oder hohen Fraktionalisierung. ${ }^{11}$

\section{Zusammenfassung}

Diese Untersuchung hatte die Verbindung von Kultur und Konflikt zum Gegenstand. Eingangs wurden dazu kulturelle Konflikte als jene Untergruppe der politischen Auseinandersetzungen definiert, in denen die Identität der beteiligten Akteure - verstanden als Referenz auf Sprache, Religion oder historische Bezüge - Thema (aber nicht unbedingt auch Ursache) des Konflikts ist. Daran anschließend wurde für den Untersuchungszeitraum 1950 bis 2005 diese Verbindung aus zwei Perspektiven beleuchtet: Zum einen wurde allgemein gefragt, wie das kulturelle setup eines Landes, gemessen sowohl als dessen religiöse und sprachliche Fraktionalisierung als auch als Durchschnitt beider Größen, dessen Konfliktrisiko beeinflusst. Zum anderen wurde spezifisch untersucht, welche der in der Konfliktforschung diskutierten Ansätze eine signifikante Erklärungsleistung für das Risiko eines kulturellen Konflikts liefern können. Angesichts theoretisch unerwarteter Ergebnisse wurde die Ausgangsthese modifiziert und es wurde erfolgreich auf eine nichtlineare Verbindung von kultureller Struktur und Konflikt getestet.

Kulturelle Konflikte, so das Ergebnis des deskriptiven Überblicks, sind vorwiegend innerstaatliche Phänomene. Zugleich sind sie sichtbar gewalttätiger als Auseinandersetzungen ohne Identitätsbezug. In der näheren statistischen Analyse konnte eine stabile positive Verbindung zwischen dem Grad sprachlicher Fraktionalisierung und dem Konfliktrisiko eines Landes isoliert werden. Dieser Bezug ist unabhängig von der Konfliktart (kulturell oder nicht-kulturell) und von den beteiligten Parteien (innerstaatliche oder zwischenstaatliche Auseinandersetzungen). Offenbar sind demnach Gesellschaften mit größerer sprachlicher Heterogenität eher von Konflikten betroffen als sprachlich homogene Länder. Die weitere Qualifizierung dieser Verbindung ergab zudem einen signifikanten Konditionaleffekt, der darauf hindeutet, dass vor allem ein mittleres Fraktionalisierungsniveau und damit eine Situation, in der sich annähernd gleich große soziale Gruppen gegenüberstehen, entscheidend sein könnte.

Für die religiöse Fraktionalisierung ergab sich hingegen eine unerwartete, signifikant negative Verbindung zwischen Fraktionalisierung und Konfliktrisiko. Nur für das allgemeine Konfliktrisiko zwischen Staaten zeigte sich das eigentlich für innerstaatliche Auseinandersetzungen vermutete positive Vorzeichen. Da Religion als Merkmal allerdings auch geleugnet werden kann und damit nur schwer erfassbar ist,

11 Hierzu sei allerdings angemerkt, dass der Befund teilweise durch die Definition der abhängigen Variable bedingt scheint: Berücksichtigt man nicht Konflikte der CONIS-Stufen 3 (»Krise «), 4 (»Begrenzter Krieg «) und 5 (»Krieg «), sondern nur (aus Platzgründen hier nicht aufgeführt) die gewalttätigeren Auseinandersetzungen der Stufen 4 und 5, ist der Interaktionsterm in den Modellen für sprachliche Fraktionalisierung positiv (wenn auch nur für Kulturkonflikte signifikant). Für die Modelle zur religiösen Fraktionalisierung bleibt er dagegen weiter negativ. 
kann dieser Befund auch durch die heterogene Datengrundlage der Studie begünstigt sein. Für eine solche Interferenz spricht, dass eine nichtlineare Spezifizierung der Regressionsmodelle einen stabilen, signifikant konfliktsteigernden Konditionaleffekt mittlerer Fraktionalisierung auf das Konfliktrisiko eines Landes auswies. Sprachliche und religiöse Fraktionalisierung zeigten zudem eine wechselseitig hemmende Verbindung zur konfliktsteigernden Wirkung des Anteils junger Männer in einer Gesellschaft, deren weitere Untersuchung lohnenswert erscheint.

Im Hinblick auf die Determinanten kultureller Konflikte und damit auf die zweite, spezifische Perspektive, ergab die Untersuchung keine funktionalen Unterschiede zwischen dem allgemeinen Konfliktrisiko eines Landes und der Wahrscheinlichkeit, in einen innerstaatlichen kulturellen Konflikt verwickelt zu sein. Die Verfügbarkeit von Ackerland, ökonomisches Wachstum, Migration und der Anteil junger Männer erwiesen sich gemessen an ihren Regressionskoeffizienten und an ihrem Signifikanzniveau insgesamt als schwächere Prädiktoren für eine kulturelle Auseinandersetzung als allgemein für das Konfliktrisiko. Umgekehrt zeigte sich die Einbindung in den Weltmarkt als signifikant positiv mit dem Risiko kulturell konnotierter Auseinandersetzungen verbunden. Für die kulturellen Einflussgrößen selbst blieb die Assoziation bis auf die oben erwähnte nichtlineare Spezifizierung ebenfalls bestehen. Auf zwischenstaatlicher Ebene erwies sich hingegen die Migrationsrate als signifikanter Prädiktor für das Risiko eines kulturellen Konflikts - eine Beziehung, die für das allgemeine Konfliktrisiko nicht nachgewiesen werden konnte.

Zusammenfassend lässt sich damit eine spürbare Wirkung kultureller Einflussgrößen auf das Konfliktrisiko von Ländern nachweisen, besonders jedoch von sprachlicher Fraktionalisierung. Die getrennte Untersuchung von inner- und zwischenstaatlichen Konflikten hat zudem gezeigt, dass Wirkungszusammenhänge jeweils unterschiedlich verlaufen. Diese Ergebnisse sind wichtige Impulse für eine differenziertere Diskussion über den Zusammenhang von Kulturen und Konfliktrisiken und können sich auch in überarbeiteten Modellen für die Konfliktfrühwarnung niederschlagen. Allerdings beeinflussen eine Reihe weitere Größen ebenfalls die Wahrscheinlichkeit einer Auseinandersetzung. Beispielsweise erhöhen die Einwohnerstärke eines Landes, ein hoher Anteil junger Männer, geringe agrarische Nutzflächen und ein geringes Wirtschaftswachstum ebenfalls die Konfliktwahrscheinlichkeit. Sprachliche und religiöse Fraktionalisierung sind damit zwar Größen, die bei der Abschätzung von Konfliktrisiken auf jeden Fall nicht ignoriert werden sollten. Da sie aber keine Erklärung »im Alleingang « leisten können, sollten sie nicht außerhalb des übrigen Kontexts betrachtet werden.

Notwendig für die ertragreiche Forschung in diesem Bereich ist folglich die Berücksichtigung des strukturellen Rahmens, in dem sich kulturelle Konflikte als kommunikative Interaktionsprozesse abspielen, und eine Gesamtschau des Zusammenspiels der einzelnen Faktoren, um den Zusammenhang zwischen Kultur und Konflikt erhellen zu können. Weiterführende Forschungsdesiderata lassen sich an dieser Stelle ausmachen, insbesondere eine prozessorientierte und dynamisierte, also konfliktphasenbezogene Konflikttypenzuordnung - Stichworte sind hier die Kulturalisierung und Ökonomisierung von Konflikten - wie auch das Forschungsfeld der Frühwarnung in 
Bezug auf kulturelle Konflikte. Gerade die CONIS-Datenbank mit ihrem Fokus auf Konfliktdynamiken und Ereignisdaten kann hier einen Beitrag leisten.

\section{Literatur}

Alesina, Alberto/Devleeschauwer, Arnaud/Easterly, William/Kurlat, Sergio/Wacziarg, Romain 2003: Fractionalization, in: Journal of Economic Growth 8: 2, 155-194.

Andreß, Hans-Jürgen/Hagenaars, Jacques/Kühnel, Steffen 1997: Analyse von Tabellen und kategorialen Daten. Log-lineare Modelle, latente Klassenanalyse, logistische Regression und GSK-Ansatz, Berlin.

Barbieri, Katherine/Reuveny, Rafael 2005: Economic Globalization and Civil War, in: The Journal of Politics 67: 4, 1228-1247.

Billington, Rosamund/Strawbridge, Sheelagh/Greensides, Leonre/Fitzsimons, Annette 1991: Culture and Society: A Sociology of Culture, Houndmills.

Collier, Paul/Hoeffler, Anke 1998: On Economic Causes of Civil War, in: Oxford Economic Papers 50: 4, 563-573.

Collier, Paul/Hoeffler, Anke 2004: Greed and Grievance in Civil War, in: Oxford Economic Papers 56: 4, 563-595.

De Juan, Alexander/Hasenclever, Andreas 2008: Framing Religious Conflicts. Die Rolle von Eliten in religiös konnotierten Gewaltkonflikten. (Paper, vorgestellt auf der Autorenkonferenz »Identität, Institutionen und Ökonomie: Ursachen innenpolitischer Gewalt«, Konstanz, 22.-23. Februar 2008).

Ellingsen, Tanja 2000: Colorful Community or Ethnic Witches' Brew? Multiethnicity and Domestic Conflict During and After the Cold War, in: Journal of Conflict Resolution 44: 2, 228-249.

Esser, Hartmut 1999: Soziologie. Spezielle Grundlagen, Band 1: Situationslogik und Handeln, Frankfurt am Main.

Fearon, James/Laitin, David 2003: Ethnicity, Insurgency, and Civil War, in: American Political Science Review 97: 1, 75-90.

Fjelde, Hanne 2008: Is There an Autocratic Civil Peace? Authoritarian Regimes and Civil War, 1971-2001 (Paper, vorgestellt beim 49th Annual Meeting der International Studies Association, San Francisco, 26.-29. März 2008), in: http://www.allacademic.com/meta/ p251458_index.html; 2.3.2009.

Fox, Jonathan/Sandler, Shmuel 2006: Religion in World Conflict, London.

Fuller, Gary 1995: The Demographic Backdrop to Ethnic Conflict: A Geographic Overview, in: Central Intelligence Agency (Hrsg.): The Challenge of Ethnic Conflict to National and International Order in the 1990s: Geographic Perspectives. A Conference Report, Washington, DC, 151-154.

Fuller, Gary/Pitts, Forrest 1990: Youth Cohorts and Political Unrest in South Korea, in: Political Geography Quarterly 9: 1, 9-22.

Fuller, Graham 2004: The Youth Crisis in Middle Eastern Society (Brief Paper April 2004, Institute for Social Policy and Understanding), in: http://ispu.org/reports/articledetailpb-62.html; 2.3.2009.

Gartzke, E. /Gleditsch, K. S. 2006: Identity and Conflict, Ties That Bind and Differences That Divide, in: European Journal of International Relations 12: 1, 53-87.

Geertz, Clifford 1994: Dichte Beschreibung. Beiträge zum Verstehen kultureller Systeme, Frankfurt am Main.

Gleditsch, Nils 1995: Democracy and the Future of European Peace, in: European Journal of International Relations 1: 4, 539-571. 
Gleditsch, Nils Petter/Wallensteen, Peter/Erikssom, Mikael/Sollenberg, Margareta/Strand, Håvard 2002: Armed Conflicts 1946-2001: A New Dataset, in: Journal of Peace Research 39: 5, 615-637.

Goffman, Erving 1977: Rahmen-Analyse. Ein Versuch über die Organisation von Alltagserfahrungen, Frankfurt am Main.

Goldstone, Jack 1991: Revolution and Rebellion in the Early Modern World, Berkeley, CA.

Goldstone, Jack/Gurr, Ted Robert/Harff, Barbara/Levy, Marc/Marshall, Monty/Bates, Robert/ Epstein, David/Kahl, Colin/Surko, Pamela/Ulfelder, John/Unger, Alan 2000: State Failure Task Force Report: Phase III Findings, in: http://globalpolicy.gmu.edu/pitf/SFTF\%20Phase\%20III\%20Report\%20Final.pdf; 1.5.2009.

Gordon, Raymond G., Jr. (Hrsg.) 2005: Ethnologue: Languages of the World, Dallas, 15. Ausgabe, in: http://www.ethnologue.com/ethno_docs/distribution.asp?by=country; 22.7.2007.

Greene, William 1993: Econometric Analysis, 2. Auflage, Upper Saddle River, NJ.

Gurr, Ted R. 1968: A Causal Model of Civil Strife: A Comparative Analysis Using New Indices, in: American Political Science Review 62: 4, 1104-1124.

Gurr, Ted R. 1970: Why Men Rebel, Princeton, NJ.

Hansen, Klaus 1993: Einleitung, in: Hansen, Klaus (Hrsg.): Kulturbegriff und Methode. Der stille Paradigmenwechsel in den Geisteswissenschaften, Tübingen, 7-15.

Hansen, Klaus, 2000: Kultur und Kulturwissenschaft. Eine Einführung, Tübingen, Basel.

Hegre, Håvard/Ellingsen, Tanja/Gates, Scott/Gleditsch, Nils Petter 2001: Toward a Democratic Civil Peace? Democracy, Political Change, and Civil War, 1816-1992, in: American Political Science Review 95: 1, 33-48.

Hegre, Håvard/Sambanis, Nicholas 2006: Sensitivity Analysis of Empirical Results on Civil War Onset, in: Journal of Conflict Resolution 50: 4, 508-535.

Heinsohn, Gunnar 2003: Söhne und Weltmacht. Terror im Aufstieg und Fall der Nationen, Zürich.

Held, David/McGrew, Anthony/Goldblatt, David/Perraton, Jonathan 1999: Global Transformations: Politics, Economics and Culture, Cambridge.

Henderson, Erol A./Tucker, R. 2001: Clear and Present Strangers: The Clash of Civilizations and International Conflict, in: International Studies Quarterly 45: 2, 317-338.

Henderson, Errol/Singer, David 2000: Civil War in the Post-Colonial World, 1946-92, in: Journal of Peace Research 37: 3, 257-299.

Heston, Alan/Summers, Robert/Aten, Bettina 2006: Penn World Table: Version 6.2, Center for International Comparisons of Production, Income and Prices at the University of Pennsylvania, September 2006, in: http://pwt.econ.upenn.edu/php_site/pwt_index.php; 2.3.2009.

Horowitz, Donald 1985: Ethnic Groups in Conflict, Berkeley, CA.

Huntington, Samuel P. 1993: The Clash of Civilizations?, in: Foreign Affairs 72: 3, 22-49.

Huntington, Samuel P. 1997: Kampf der Kulturen. Die Neugestaltung der Weltpolitik im 21. Jahrhundert, München.

Jaggers, Keith/Gurr, Robert 1995: Transitions to Democracy: Tracking Democracy's Third Wave with the Polity III Data, in: Journal of Peace Research 32: 4, 469-482.

Krallmann, Dieter/Ziemann, Andreas 2001: Grundkurs Kommunikationswissenschaft. München.

Liao, Tim 1994: Interpreting Probability Models: Logit, Probit, and Other Generalized Linear Models, Thousand Oaks, CA.

Luhmann, Niklas 1984: Soziale Systeme. Grundriß einer allgemeinen Theorie, 1. Auflage, Frankfurt am Main.

Luhmann, Niklas 1985: Soziale Systeme. Grundriß einer allgemeinen Theorie, 2. Auflage, Frankfurt am Main. 
Marshall, Monty/Jaggers, Keith 2005: Polity IV Project, Political Regime Characteristics and Transitions 1800-2004, Version p4v 2004, in: http://www.systemicpeace.org/polity/polity4.htm; 2.3.2009.

Martin, Philippe/Thoenig, Mathias/Mayer, Thierry 2008: Civil Wars and International Trade, in: Journal of the European Economic Association 6: 2-3, 541-550.

McDonald, Patrick 2004: Peace Through Trade or Free Trade?, in: Journal of Conflict Resolution 48: 4, 547-572.

Mesquida, Christian/Wiener, Neil 1999: Male Age Composition and Severity of Conflicts, in: Politics and the Life Sciences 18: 2, 181-189.

Messmer, Heinz 2003: Der soziale Konflikt. Kommunikative Emergenz und systemische Reproduktion, Stuttgart.

Mintzel, Alf 1993: Kultur und Gesellschaft. Der Kulturbegriff in der Soziologie, in: Hansen, Klaus (Hrsg.): Kulturbegriff und Methode. Der stille Paradigmenwechsel in den Geisteswissenschaften, Tübingen, 171-201.

Montalvo, José/Reynal-Querol, Marta 2005: Ethnic Polarization, Potential Conflict, and Civil Wars, in: The American Economic Review 95: 3, 796-816.

Muller, Edward/Weede, Erich 1990: Cross-National Variation in Political Violence: A Rational Action Approach, in: Journal of Conflict Resolution 34: 4, 624-651.

Oneal, John/Russett, Bruce 1999: Assessing the Liberal Peace With Alternative Specifications: Trade Still Reduces Conflict, in: Journal of Peace Research 36: 4, 423-442.

Parsons, Talcott 1978: A Paradigm of the Human Condition, in: Parsons, Talcott (Hrsg.): Action Theory and the Human Condition, New York, NY, 352-433.

Parsons, Talcott/Shils, Edward 1990: Values and Social Systems, in: Alexander, Jeffrey/Seidman, Steven (Hrsg.): Culture and Society: Contemporary Debates, Cambridge, 39-46.

Polachek, Solomon 1997: Why Democracies Cooperate More and Fight Less: The Relationship between International Trade and Cooperation, in: Review of International Economics 5: 3, 295-309.

Rosecrance, Richard 1986: The Rise of the Trading State: Commerce and Conquest in the Modern World, New York, NY.

Russett, Bruce/Oneal, John R./Cox, M. 2000: Clash of Civilizations or Realism and Liberalism déjà-vu? Some Evidence, in: Journal of Peace Research 37: 5, 583-608.

Sarkees, Meredith Reid 2000: Correlates of War Data on War: An Update to 1997, in: Conflict Management and Peace Science 18: 1, 123-144.

Schmid, Michael 1992: The Concept of Culture and Its Place within a Theory of Social Action, in: Münch, Richard/Smelser, Neil (Hrsg.): Theory of Culture, Berkeley, CA, 88-120.

Schwank, Nicolas 2010: Konflikte, Krisen, Kriege. Merkmale und Dynamiken internationaler politischer Konflikte 1945-2005, Baden-Baden.

Schwelling, Birgit 2004: Der kulturelle Blick auf politische Phänomene. Theorien, Methoden, Problemstellungen, in: Schwelling, Birgit (Hrsg.): Politikwissenschaft als Kulturwissenschaft. Theorien, Methoden, Problemstellungen, Wiesbaden, 11-29.

Sen, Amartya 1998: Mortality as an Indicator of Economic Success and Failure, in: The Economic Journal 108: 446, 1-25.

Seul, Jeffrey 1999: »Ours is the Way of God«: Religion, Identity, and Intergroup Conflict, in: Journal of Peace Research 36: 5, 553-569.

Singer, David/Small, Melvin 1972: The Wages of War 1816-1965: A Statistical Handbook, New York, NY.

Small, Melvin/Singer David J. 1982: Resort to Arms: International and Civil Wars, 1816-1980, Beverly Hills, CA.

Smelser, Neil 1992: Culture: Coherent or Incoherent, in: Münch, Richard/Smelser, Neil (Hrsg): Theory of Culture, Berkeley, CA, 3-28.

Strand, Håvard 2007: Retreating From a Civil Democratic Peace? Revisiting the Relationship between Political Institutions and Civil War (Research Note, Political Science Department, 
University of Oslo, Center for the Study of Civil War, PRIO), in: http://www.prio.no/files/ manual-import/strand_2007.pdf; 2.3.2009.

United Nations Population Division 2005: World Population Prospects: The 2004 Revision, Extended dataset, New York, NY.

Uppsala Conflict Data Program (UCDP) 2010: Armed Conflict Dataset v. 4-2008 (1946-2007), in: www.ucdp.uu.se/database; 10.01.2010.

Urban, Dieter/Mayerl, Jochen 2006: Regressionsanalyse. Theorie, Technik und Anwendung, Wiesbaden.

Urdal, Henrik 2004: The Devil in the Demographics: The Effect of Youth Bulges on Domestic Armed Conflict, 1950-2000 (The World Bank, Conflict Prevention and Reconstruction Unit: Social Development Working Paper 14), in: http://www-wds.worldbank.org/external/default/WDSContentServer/WDSP/IB/2004/07/28/000012009_20040728162225/ Rendered/PDF/29740.pdf; 2.3.2009.

Urdal, Henrik 2006: A Clash of Generations? Youth Bulges and Political Violence, in: International Studies Quarterly 50: 3, 607-629.

Wagschal, Uwe/Metz, Thomas/Schwank, Nicolas 2008: Ein »demografischer Frieden«? Der Einfluss von Bevölkerungsfaktoren auf inner- und zwischenstaatliche Konflikte, in: Zeitschrift für Politikwissenschaft 18: 3, 353-383.

World Bank 2005: The World Development Indicators CD-ROM, Washington, DC. 